\title{
Dissections, orientations, and trees, with applications to optimal mesh encoding and to random sampling
}

\author{
ÉRIC FUSY, DOMINIQUE POULALHON and GILLES SCHAEFFER \\ É.F and G.S: LIX, École Polytechnique. D.P: Liafa, Univ. Paris 7. France
}

\begin{abstract}
We present a bijection between some quadrangular dissections of an hexagon and unrooted binary trees, with interesting consequences for enumeration, mesh compression and graph sampling.

Our bijection yields an efficient uniform random sampler for 3-connected planar graphs, which turns out to be determinant for the quadratic complexity of the current best known uniform random sampler for labelled planar graphs [Fusy, Analysis of Algorithms 2005].

It also provides an encoding for the set $\mathcal{P}(n)$ of $n$-edge 3 -connected planar graphs that matches the entropy bound $\frac{1}{n} \log _{2}|\mathcal{P}(n)|=2+o(1)$ bits per edge (bpe). This solves a theoretical problem recently raised in mesh compression, as these graphs abstract the combinatorial part of meshes with spherical topology. We also achieve the optimal parametric rate $\frac{1}{n} \log _{2}|\mathcal{P}(n, i, j)|$ bpe for graphs of $\mathcal{P}(n)$ with $i$ vertices and $j$ faces, matching in particular the optimal rate for triangulations.

Our encoding relies on a linear time algorithm to compute an orientation associated to the minimal Schnyder wood of a 3-connected planar map. This algorithm is of independent interest, and it is for instance a key ingredient in a recent straight line drawing algorithm for 3-connected planar graphs [Bonichon et al., Graph Drawing 2005].

Categories and Subject Descriptors: G.2.1 [Discrete Mathematics]: Combinatorial algorithms General Terms: Algorithms

Additional Key Words and Phrases: Bijection, Counting, Coding, Random generation
\end{abstract}

\section{INTRODUCTION}

One origin of this work can be traced back to an article of Ed Bender in the American Mathematical Monthly [Bender 1987], where he asked for a simple explanation of the remarkable asymptotic formula

$$
|\mathcal{P}(n, i, j)| \sim \frac{1}{3^{5} 2^{4} i j n}\left(\begin{array}{c}
2 i-2 \\
j+2
\end{array}\right)\left(\begin{array}{c}
2 j-2 \\
i+2
\end{array}\right)
$$

for the cardinality of the set of 3-connected (unlabelled) planar graphs with $i$ vertices, $j$ faces and $n=i+j-2$ edges, $n$ going to infinity. By a theorem of Whitney [1933], these graphs have essentially a unique embedding on the sphere up to homeomorphisms, so that their study amounts to that of rooted 3-connected maps, where a map is a graph embedded in the plane and rooted means with a marked oriented edge.

\subsection{Graphs, dissections and trees}

Another known property of 3-connected planar graphs with $n$ edges is the fact that they are in direct one-to-one correspondence with dissections of the sphere into $n$ quadrangles that have no non-facial 4-cycle. The heart of our paper lies in a further one-to-one correspondence. 
THEOREM 1.1. There is a one-to-one correspondence between unrooted binary trees with $n$ nodes and unrooted quadrangular dissections of an hexagon with $n$ interior vertices and no non-facial 4-cycle.

The mapping from binary trees to dissections, which we call the closure, is easily described and resembles constructions that were recently proposed for simpler kinds of maps [Schaeffer 1997; Bouttier et al. 2002; Poulalhon and Schaeffer 2006]. The proof that the mapping is a bijection is instead rather sophisticated, relying on new properties of constrained orientations [Ossona de Mendez 1994], related to Schnyder woods of triangulations and 3-connected planar maps [Schnyder 1990; di Battista et al. 1999; Felsner 2001] .

Conversely, the reconstruction of the tree from the dissection relies on a linear time algorithm to compute the minimal Schnyder woods of a 3-connected map (or equivalently, the minimal $\alpha_{0}$-orientation of the associated derived map, see Section 9). This problem is of independant interest and our algorithm has for example applications in the graph drawing context [Bonichon et al. 2007]. It is akin to Kant's canonical ordering [Kant 1996; Chuang et al. 1998; Bonichon et al. 2003; Castelli-Aleardi and Devillers 2004], but again the proof of correctness is quite involved.

Theorem 1.1 leads directly to the implicit representation of the numbers $\left|\mathcal{P}_{n}^{\prime}\right|$ - counting rooted 3-connected maps with $n$ edges - due to Tutte [1963]), and its refinement as discussed in Section 5 yields that of $\left|\mathcal{P}_{i j}^{\prime}\right|$ the number of rooted 3connected maps with $i$ vertices and $j$ faces (due to Mullin and Schellenberg [1968]) from which Formula (1) follows. It partially explains the combinatorics of the occurrence of the cross product of binomials, since these are typical of binary tree enumerations. Let us mention that the one-to-one correspondence specializes particularly nicely to count plane triangulations (i.e., 3-connected maps with all faces of degree 3), leading to the first bijective derivation of the counting formula for unrooted plane triangulations with $i$ vertices, originally found by Brown [1964] using algebraic methods.

\subsection{Random sampling}

A second byproduct of Theorem 1.1 is an efficient uniform random sampler for rooted 3-connected maps, i.e., an algorithm that, given $n$, outputs a random element in the set $\mathcal{P}_{n}^{\prime}$ of rooted 3-connected maps with $n$ edges with equal chances for all elements. The same principles yield a uniform sampler for $\mathcal{P}_{i j}^{\prime}$.

The uniform random generation of classes of maps like triangulations or 3connected graphs was first considered in mathematical physics (see references in [Ambjørn et al. 1994; Poulalhon and Schaeffer 2006]), and various types of random planar graphs are commonly used for testing graph drawing algorithms (see [de Fraysseix et al.]).

The best previously known algorithm [Schaeffer 1999] had expected complexity $O\left(n^{5 / 3}\right)$ for $\mathcal{P}_{n}^{\prime}$, and was much less efficient for $\mathcal{P}_{i j}^{\prime}$, having even exponential complexity for $i / j$ or $j / i$ tending to 2 (due to Euler's formula these ratio are bounded above by 2 for 3 -connected maps). In Section 6 , we show that our generator for $\mathcal{P}_{n}^{\prime}$ or $\mathcal{P}_{i j}^{\prime}$ performs in linear time except if $i / j$ or $j / i$ tends to 2 where it becomes at most cubic.

ACM Journal Name, Vol. V, No. N, Month 20YY. 
From the theoretical point of view, it is also desirable to work with the uniform distribution on planar graphs. However, random (labelled) planar graphs appear to be challenging mathematical objects [Osthus et al. 2003; McDiarmid et al. 2005]. A Markov chain converging to the uniform distribution on planar graphs with $i$ vertices was given by Denise et al. [1996], but it resists known approaches for perfect sampling [Wilson 2004], and has unknown mixing time. As opposed to this, a recursive scheme to sample planar graphs was proposed by Bodirsky et al. [2003], with amortized complexity $O\left(n^{6.5}\right)$. This result is based on a recursive decomposition of planar graphs: a planar graph can be decomposed into a tree-structure whose nodes are occupied by rooted 3-connected maps. Generating a planar graph reduces to computing branching probabilities so as to generate the decomposition tree with suitable probability; then a random rooted 3-connected map is generated for each node of the decomposition tree. Bodirsky et al. [2003] use the so-called recursive method [Nijenhuis and Wilf 1978; Flajolet et al. 1994; Wilson 1997] to take advantage of the recursive decomposition of planar graphs. Our new random generator for rooted 3-connected maps reduces their amortized cost to $O\left(n^{3}\right)$. Finally a new uniform random generator for planar graphs was recently developped by one of the authors [Fusy 2005], that avoids the expensive preprocessing computations of [Bodirsky et al. 2003]. The recursive scheme is similar to the one used in [Bodirsky et al. 2003], but the method to translate it to a random generator relies on Boltzmann samplers, a new general framework for the random generation recently developed in [Duchon et al. 2004]. Thanks to our random generator for rooted 3-connected maps, the algorithm of [Fusy 2005] has a time-complexity of $\mathcal{O}\left(n^{2}\right)$ for exact size uniform sampling and even performs in linear time for approximate size uniform sampling.

\subsection{Succinct encoding}

A third byproduct of Theorem 1.1 is the possibility to encode in linear time a 3 connected planar graph with $n$ edges by a binary tree with $n$ nodes. In turn the tree can be encoded by a balanced parenthesis word of $2 n$ bits. This code is optimal in the information theoretic sense: the entropy per edge of this class of graphs, i.e., the quantity $\frac{1}{n} \log _{2}|\mathcal{P}(n)|$, tends to 2 when $n$ goes to infinity, so that a code for $\mathcal{P}(n)$ cannot give a better guarantee on the compression rate.

Applications calling for compact storage and fast transmission of 3D geometrical meshes have recently motivated a huge literature on compression, in particular for the combinatorial part of the meshes. The first compression algorithms dealt only with triangular faces [Rossignac 1999; Touma and Gotsman 1998], but many meshes include larger faces, so that polygonal meshes have become prominent (see [Alliez and Gotsman 2003] for a recent survey).

The question of optimality of coders was raised in relation with exception codes produced by several heuristics when dealing with meshes with spherical topology [Gotsman 2003; Khodakovsky et al. 2002]. Since these meshes are exactly triangulations (for triangular meshes) and 3-connected planar graphs (for polyhedral ones), the coders in [Poulalhon and Schaeffer 2006] and in the present paper respectively prove that traversal based algorithms can achieve optimality.

On the other hand, in the context of succinct data structures, almost optimal algorithms have been proposed [He et al. 2000; Lu 2002], that are based on separator 
theorems. However these algorithms are not truly optimal (they get $\varepsilon$ close to the entropy but at the cost of an uncontrolled increase of the constants in the linear complexity). Moreover, although they rely on a sophisticated recursive structure, they do not support efficient adjacency requests.

As opposed to that, our algorithm shares with [He et al. 1999; Bonichon et al. 2003 the property that it produces essentially the code of a spanning tree. More precisely it is just the balanced parenthesis code of a binary tree, and adjacencies of the initial dissection that are not present in the tree can be recovered from the code by a simple variation on the interpretation of the symbols. Adjacency queries can thus be dealt with in time proportional to the degree of vertices [Castelli-Aleardi et al. 2006] using the approach of [Munro and Raman 1997; He et al. 1999].

Finally we show that the code can be modified to be optimal on the class $\mathcal{P}(n, i, j)$. Since the entropy of this class is strictly smaller than that of $\mathcal{P}(n)$ as soon as $|i-n / 2| \gg n^{1 / 2}$, the resulting parametric coder is more efficient in this range. In particular in the case $j=2 i-4$ our new algorithm specializes to an optimal coder for triangulations.

\subsection{Outline of the paper}

The paper starts with two sections of preliminaries: definitions of the maps and trees involved (Section 2), and some basic correspondences between them (Section 3). Then comes our main result (Section 4), the mapping between binary trees and some dissections of the hexagon by quadrangular faces. The fact that this mapping is a bijection follows from the existence and uniqueness of a certain tri-orientation of our dissections. The proof of this auxiliary theorem, which requires the introduction of the so-called derived maps and their $\alpha_{0}$-orientations, is delayed to Section 8 , that is, after the three sections dedicated to applications of our main result: in these sections we successively discuss counting (Section 5), sampling (Section 6) and coding (Section 7) rooted 3-connected maps. The third application leads us to our second important result: in Section 9 we present a linear time algorithm to compute the minimal $\alpha_{0}$-orientation of the derived map of a 3-connected planar map (which also corresponds to the minimal Schnyder woods alluded to above). Finally, Section 10 is dedicated to the correctness proof of this orientation algorithm. Figure 1 summarizes the connections between the different families of objects we consider.

\section{DEFINITIONS}

\subsection{Planar maps}

A planar map is a proper embedding of an unlabelled connected graph in the plane, where proper means that edges are smooth simple arcs that do not meet but at their endpoints. A planar map is said to be rooted if one edge of the outer face, called the root-edge, is marked and oriented such that the outer face lays on its right. The origin of the root-edge is called root-vertex. Vertices and edges are said to be outer or inner depending on whether they are incident to the outer face or not.

A planar map is 3-connected if it has at least 4 edges and can not be disconnected by the removal of two vertices. The first 3-connected planar map is the tetrahedron,

ACM Journal Name, Vol. V, No. N, Month 20YY. 


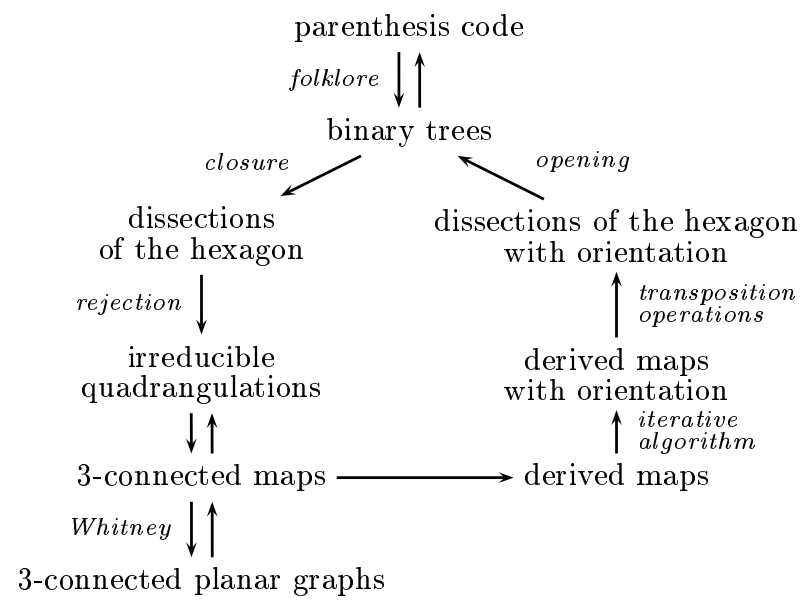

Fig. 1. Relations between involved objects.

which has 6 edges. We denote by $\mathcal{P}_{n}^{\prime}$ (respectively $\mathcal{P}_{i j}^{\prime}$ ) the set of rooted 3 -connected planar maps with $n$ edges (resp. $i$ vertices and $j$ faces). A 3-connected planar map is outer-triangular if its outer face is triangular.

\subsection{Plane trees, and half-edges}

Plane trees are planar maps with a single face - the outer one. A vertex is called a leaf if it has degree 1, and node otherwise. Edges incident to a leaf are called stems, and the other are called entire edges. Observe that plane trees are unrooted trees.

Binary trees are plane trees whose nodes have degree 3. By convention we shall require that a rooted binary tree has a root-edge that is a stem. The root-edge of a rooted binary tree thus connects a node, called the root-node, to a leaf, called the root-leaf. With this definition of rooted binary tree, upon drawing the tree in a top down manner starting with the root-leaf, every node (including the root-node) has a father, a left son and a right son. This (very minor) variation on the usual definition of rooted binary trees will be convenient later on. For $n \geq 1$, we denote respectively by $\mathcal{B}_{n}$ and $\mathcal{B}_{n}^{\prime}$ the sets of binary and rooted binary trees with $n$ nodes (they have $n+2$ leaves, as proved by induction on $n$ ). These rooted trees are well known to be counted by the Catalan numbers: $\left|\mathcal{B}_{n}^{\prime}\right|=\frac{1}{n+1}\left(\begin{array}{c}2 n \\ n\end{array}\right)$.

The vertices of a binary tree can be greedily bicolored - say in black or whiteso that adjacent vertices have distinct colors. The bicoloration is unique up to the choice of the color of the first node. As a consequence, rooted bicolored binary trees are either black-rooted or white-rooted, depending on the color of the root node. The sets of black-rooted (resp. white-rooted) binary trees with $i$ black nodes and $j$ white nodes is denoted by $\mathcal{B}_{i j}^{\bullet}$ (resp. by $\mathcal{B}_{i j}^{\circ}$ ); and the total set of rooted bicolored binary trees with $i$ black nodes and $j$ white nodes is denoted by $\mathcal{B}_{i j}^{\prime}$.

It will be convenient to view each entire edge of a tree as a pair of opposite halfedges - each one incident to one extremity of the edge - and to view each stem as a single half-edge -incident to the node holding the stem. More generally we shall 
consider maps that have entire edges (made of two half-edges) and stems (made of only one half-edge). It is then also natural to associate one face to each half-edge, say, the face on its right. In the case of trees, there is only the outer face, so that all half-edges get the same associated face.

\subsection{Quadrangulations and dissections}

A quadrangulation is a planar map whose faces (including the outer one) have degree 4. A dissection of the hexagon by quadrangular faces is a planar map whose outer face has degree 6 and inner faces have degree 4 .

Cycles that do not delimit a face are said to be separating. A quadrangulation or a dissection of the hexagon by quadrangular faces is said to be irreducible if it has at least 4 faces and has no separating 4-cycle. The first irreducible quadrangulation is the cube, which has 6 faces. We denote by $\mathcal{Q}_{n}^{\prime}$ the set of rooted irreducible quadrangulations with $n$ faces, including the outer one. Euler's relation ensures that these quadrangulations have $n+2$ vertices. We denote by $\mathcal{D}_{n}\left(\mathcal{D}_{n}^{\prime}\right)$ the set of (rooted, respectively) irreducible dissections of the hexagon with $n$ inner vertices. These have $n+2$ quadrangular faces, according to Euler's relation. From now on, irreducible dissections of the hexagon by quadrangular faces will simply be called irreducible dissections. The classes of rooted irreducible quadrangulations and of rooted irreducible dissections are respectively denoted by $\mathcal{Q}^{\prime}=\cup_{n} \mathcal{Q}_{n}^{\prime}$ and $\mathcal{D}^{\prime}=\cup_{n} \mathcal{D}_{n}^{\prime}$.

As faces of dissections and quadrangulations have even degree, the vertices of these maps can be greedily bicolored, say, in black and white, so that each edge connects a black vertex to a white one. Such a bicoloration is unique up to the choice of the colors. We denote by $\mathcal{Q}_{i j}^{\prime}$ the set of rooted bicolored irreducible quadrangulations with $i$ black vertices and $j$ white vertices and such that the rootvertex is black; and by $\mathcal{D}_{i j}^{\prime}$ the set of rooted bicolored irreducible dissections with $i$ black inner vertices and $j$ white inner vertices and such that the root-vertex is black.

A bicolored irreducible dissection is complete if the three outer white vertices of the hexagon have degree exactly 2 . Hence, these three vertices are incident to two adjacent edges on the hexagon.

\section{CORRESPONDENCES BETWEEN FAMILIES OF PLANAR MAPS}

This section recalls a folklore bijection between irreducible quadrangulations and 3-connected maps, hereafter called angular mapping, see [Mullin and Schellenberg 1968], and its adaptation to outer-triangular 3-connected maps.

\subsection{3-connected maps and irreducible quadrangulations}

Let us first recall how the angular mapping works. Given a rooted quadrangulation $Q \in \mathcal{Q}_{n}^{\prime}$ endowed with its vertex bicoloration, let $M$ be the rooted map obtained by linking, for each face $f$ of $Q$ (even the outer face), the two diagonally opposed black vertices of $f$; the root of $M$ is chosen to be the edge corresponding to the outer face of $Q$, oriented so that $M$ and $Q$ have same root-vertex, see Figure 2. The map $M$ is often called the primal map of $Q$. A similar construction using white vertices instead of black ones would give its dual map (i.e., the map with a vertex 


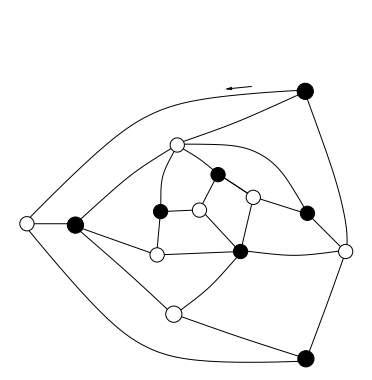

(a) A quadrangulation

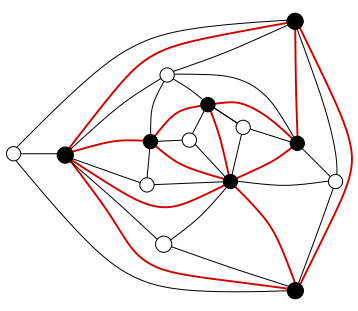

(b) with its black diagonals

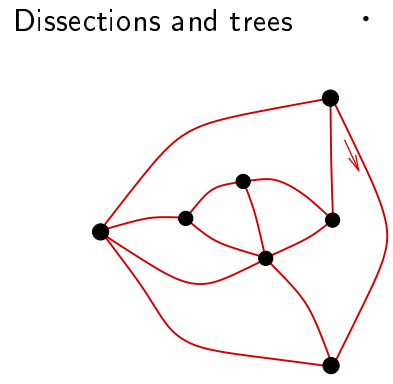

(c) gives a planar map.

Fig. 2. The angular mapping: from a rooted irreducible quadrangulation to a rooted 3-connected planar map.

in each face of $M$ and edge-set corresponding to the adjacencies between vertices and faces of $M$ ).

The construction of the primal map is easily invertible. Given any rooted map $M$, the inverse construction consists in adding a vertex called a face-vertex in each face (even the outer one) of $M$ and linking a vertex $v$ and a face-vertex $v_{f}$ by an edge if $v$ is incident to the face $f$ corresponding to $v_{f}$. Keeping only these facevertex incidence edges yields a quadrangulation. The root is chosen as the edge that follows the root of $M$ in counter-clockwise order around its origin.

The following theorem is a classical result in the theory of maps.

Theorem 3.1 (ANGUlar MAPPING). The angular mapping is a bijection between $\mathcal{P}_{n}^{\prime}$ and $\mathcal{Q}_{n}^{\prime}$ and more precisely a bijection between $\mathcal{P}_{i j}^{\prime}$ and $\mathcal{Q}_{i j}^{\prime}$.

3.2 Outer-triangular 3-connected maps and bicolored complete irreducible dissections

The same principle yields a bijection, also called angular mapping, between outertriangular 3-connected maps and bicolored complete irreducible dissections, which will prove very useful in Sections 7 and 8. This mapping is very similar to the angular mapping: given a complete dissection $D$, associate to $D$ the map $M$ obtained by linking the two black vertices of each inner face of $D$ by a new edge, see Figure 3. The map $M$ is called the primal map of $D$.

THEOREM 3.2 (ANGUlAR MAPPING WITH BORDER). The angular mapping, formulated for complete dissections, is a bijection between bicolored complete irreducible dissections with $i$ black vertices and $j$ white vertices and outer-triangular 3-connected maps with $i$ vertices and $j-3$ inner faces.

Proof. The proof follows similar lines as that of Theorem 3.1, see [Mullin and Schellenberg 1968].

\subsection{Derived maps}

In its version for complete dissections, the angular mapping can also be formulated using the concept of derived map, which will be very useful throughout this article (in particular when dealing with orientations).

Let $M$ be an outer-triangular 3-connected map, and let $M^{*}$ be the map obtained from the dual of $M$ by removing the dual vertex corresponding to the outer face of $M$. Then the derived map $M^{\prime}$ of $M$ is the superimposition of $M$ and $M^{*}$, where 


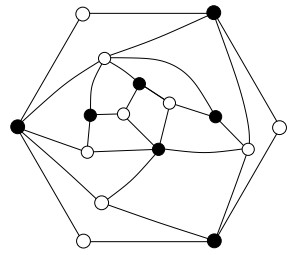

(a) A dissection,

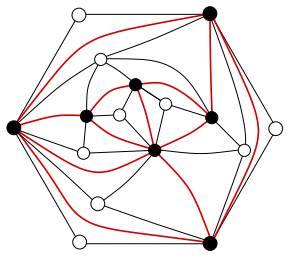

(b) black diagonals,

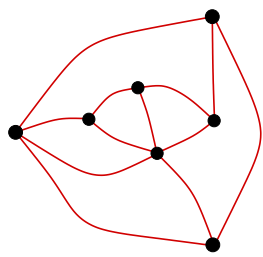

(c) the 3-connected map,

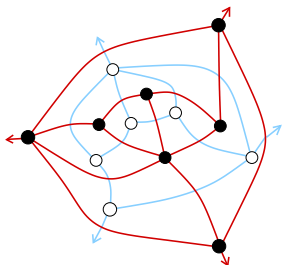

(d) the derived map.

Fig. 3. The angular mapping with border: from a bicolored complete irreducible dissection (a) to an outer-triangular 3 -connected map (c). The common derived map is shown in (d).

each outer vertex receives an additional half-edge directed toward the outer face. For example, Figure 3(d) shows the derived map of the map given in Figure 3(c). The map $M$ is called the primal map of $M^{\prime}$ and the map $M^{*}$ is called the dual map of $M^{\prime}$. Observe that the superimposition of $M$ and $M^{*}$ creates a vertex of degree 4 for each edge $e$ of $M$, due to the intersection of $e$ with its dual edge. These vertices of $M^{\prime}$ are called edge-vertices. An edge of $M^{\prime}$ either corresponds to an half-edge of $M$ when it connects an edge-vertex and a primal vertex, or to an half-edge of $M^{*}$ when it connects an edge-vertex and a dual vertex.

Similarly, one defines derived maps of complete irreducible dissections. Given a bicolored complete irreducible dissection $D$, the derived map $M^{\prime}$ of $D$ is constructed as follows; for each inner face $f$ of $D$, link the two black vertices incident to $f$ by a primal edge, and the two white ones by a dual edge. These two edges, which are the two diagonals of $f$, intersect at a new vertex called an edge-vertex. The derived map is then obtained by keeping the primal and dual edges and all vertices except the three outer white ones and their incident edges. Finally, for the sake of regularity, each of the six outer vertices of $M^{\prime}$ receives an additional half-edge directed toward the outer face. For example, the derived map of the dissection of Figure 3(a) is shown in Figure 3(d). Black vertices are called primal vertices and white vertices are called dual vertices of the derived map $M^{\prime}$. The submap $M\left(M^{*}\right)$ of $M^{\prime}$ consisting of the primal vertices and primal edges (resp. the dual vertices and dual edges) is called the primal map (resp. the dual map) of the derived map. Clearly, $M$ has a triangular outer face; and, by construction, a bicolored complete irreducible dissection and its primal map have the same derived map.

\section{BIJECTION BETWEEN BINARY TREES AND IRREDUCIBLE DISSECTIONS}

\subsection{Closure mapping: from trees to dissections}

Local and partial closure. Given a map with entire edges and stems (for instance a tree), we define a local closure operation, which is based on a counter-clockwise walk around the map: this walk alongside the boundary of the outer map visits a succession of stems and entire edges, or more precisely, a sequence of half-edges having the outer face on their right-hand side. When a stem is immediately followed in this walk by three entire edges, its local closure consists in the creation of an opposite half-edge for this stem, which is attached to farthest endpoint of the third entire edge: this amounts to completing the stem into an entire edge, so as to create - or close - a quadrangular face. This operation is illustrated in Figure 4(b).

ACM Journal Name, Vol. V, No. N, Month 20YY. 


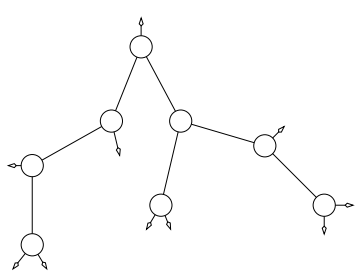

(a) A binary tree,

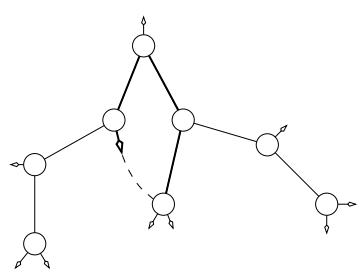

(b) a local closure,

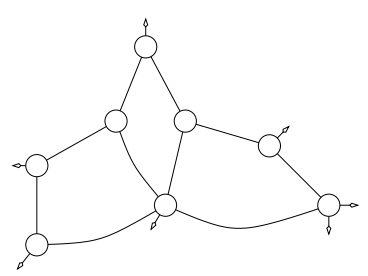

(c) and the partial closure.

Fig. 4. The partial closure.

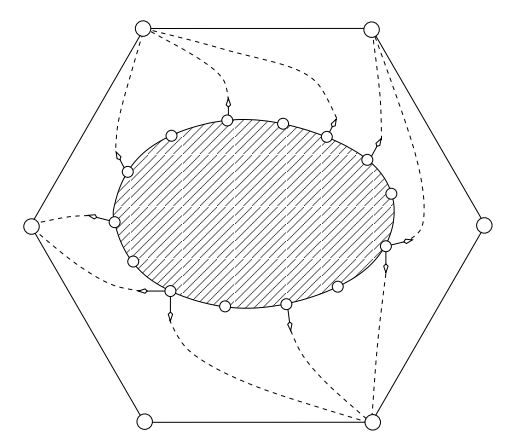

(a) Generic case when $r=2$ and $s=2$.

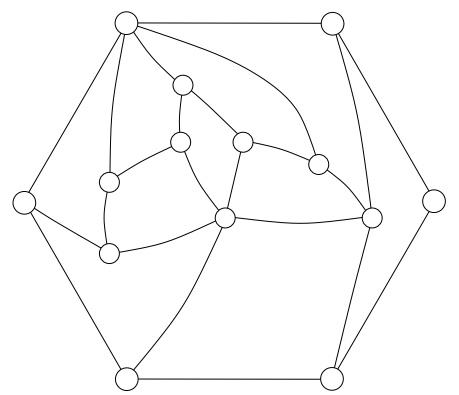

(b) Case of the binary tree of Figure 4(a).

Fig. 5. The complete closure.

Given a binary tree $T$, the local closure can be performed greedily until no more local closure is possible. Each local closure creates a new entire edge, maybe making a new local closure possible. It is easy to see that the final map, called the partial closure of $T$, does not depend on the order of the local closures. Indeed, a cyclic parenthesis word is associated to the counter-clockwise boundary of the tree, with an opening parenthesis of weight 3 for a stem and a closing parenthesis for a side of entire edge; then the future local closures correspond to matchings of the parenthesis word. An example of partial closure is shown in Figure 4(c).

Complete closure. Let us now complete the partial closure operation to obtain a dissection of the hexagon with quadrangular faces. An outer entire half-edge is an half-edge belonging to an entire edge and incident to the outer face. Observe that a binary tree $T$ with $n$ nodes has $n+2$ stems and $2 n-2$ outer entire half-edges. Each local closure decreases by 1 the number of stems and by 2 the number of outer entire half-edges. Hence, if $k$ denotes the number of (unmatched) stems in the partial closure of $T$, there are $2 k-6$ outer entire half-edges. Moreover, stems delimit intervals of inner half-edges on the contour of the outer face; these intervals have length at most 2, otherwise a local closure would be possible. Let $r$ be the number of such intervals of length 1 and $s$ be the number of such intervals of length 0 (that is, the number of nodes incident to two unmatched stems). Then $r$ and $s$ are clearly related by the relation $r+2 s=6$.

The complete closure consists in completing all unmatched stems with half-edges 


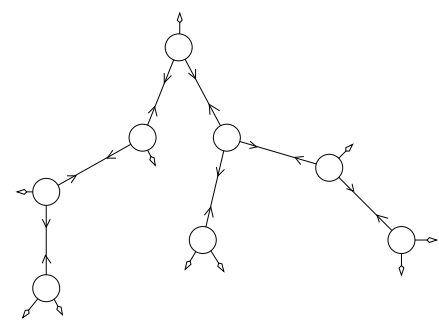

(a) A tri-oriented binary tree,

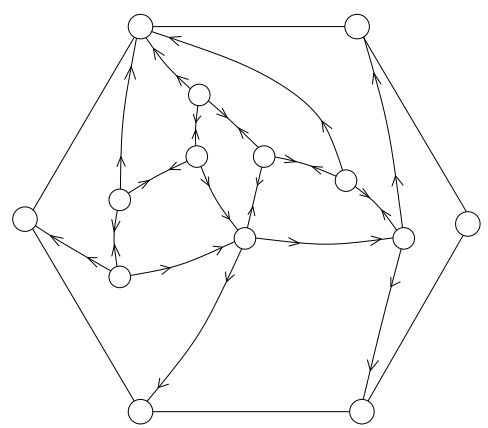

(b) and its tri-oriented closure.

Fig. 6. Examples of tri-orientations.

incident to vertices of the hexagon in the unique way (up to rotation of the hexagon) that creates only quadrangular bounded faces. Figure 5(a) illustrates the complete closure for the case $(r=2, s=2)$, and a particular example is given in Figure 5(b).

Lemma 4.1. The closure of a binary tree is an irreducible dissection of the hexagon.

Proof. Assume that there exists a separating 4-cycle $\mathcal{C}$ in the closure of $T$. Let $m \geq 1$ be the number of vertices in the interior of $\mathcal{C}$. Then there are $2 m$ edges in the interior of $\mathcal{C}$ according to Euler's relation. Let $v$ be a vertex of $T$ that belongs to the interior of $\mathcal{C}$ after the closure. Consider the orientation of edges of $T$ away from $v$ (only for the sake of this proof). Then nodes of $T$ have outdegree 2, except $v$, which has outdegree 3 . This orientation naturally induces an orientation of edges of the closure-dissection with the same property (except that vertices of the hexagon have outdegree 0 ). Hence there are at least $2 m+1$ edges in the interior of $\mathcal{C}$, a contradiction.

\subsection{Tri-orientations and opening}

Tri-orientations. In order to define the mapping inverse to the closure, we need a better description of the structure induced on the closure map by the original tree. Let us consider orientations of the half-edges of a map (in contrast to the usual notion of orientation, where edges are oriented). An half-edge is said to be inward if it is oriented toward its origin and outward if it is oriented out of its origin. If a map is endowed with an orientation of its half-edges, the outdegree of a vertex $v$ is naturally defined as the number of its incident half-edges oriented outward. The (unique) tri-orientation of a binary tree is defined as the orientation of its half-edges such that any node has outdegree 3, see Figure 6(a) for an example. A tri-orientation of a dissection is an orientation of its inner half-edges (i.e., halfedges belonging to inner edges) such that outer and inner vertices have respectively outdegree 0 and 3, and such that two half-edges of a same inner edge can not both be oriented inward, see Figure 6(b). An edge is said to be simply oriented if its two half-edges have same direction (that is, one is oriented inward and the other one outward), and bi-oriented if they are both oriented outward.

Let $D$ be an irreducible dissection endowed with a tri-orientation. A clockwise 
circuit of $D$ is a simple cycle $\mathcal{C}$ consisting of edges that are either bi-oriented or simply oriented with the interior of $\mathcal{C}$ on their right.

Lemma 4.2. Let $D$ be an irreducible dissection with $n$ inner vertices. Then a tri-orientation of $D$ has $n-1$ bi-oriented edges and $n+2$ simply oriented edges.

If a tri-orientation of a dissection has no clockwise circuit, then its bi-oriented edges form a tree spanning the inner vertices of the dissection.

Proof. Let $s$ and $r$ denote the numbers of simply and bi-oriented edges of $D$. According to Euler's relation (using the degrees of the faces), $D$ has $2 n+1$ inner edges, i.e., $2 n+1=r+s$. Moreover, as all inner vertices have outdegree 3, $3 n=2 r+s$. Hence $r=n-1$ and $s=n+2$.

If the tri-orientation has no clockwise circuit, the subgraph $H$ induced by the bioriented edges has $r=n-1$ edges, no cycle (otherwise the cycle could be traversed clockwise, as all its edges are bi-oriented), and is incident to at most $n$ vertices, which are the inner vertices of $D$. According to a classical result of graph theory, $H$ is a tree spanning the $n$ inner vertices of $D$.

Closure-tri-orientation of a dissection. Let $D$ be a dissection obtained as the closure of a binary tree $T$. The tri-orientation of $T$ clearly induces via the closure a triorientation of $D$, called closure-tri-orientation. On this tri-orientation, bi-oriented edges correspond to inner edges of the original binary tree, see Figure 6(b).

Lemma 4.3. A closure-tri-orientation has no clockwise circuit.

Proof. Since vertices of the hexagon have outdegree 0 , they can not belong to any circuit. Hence clockwise circuits may only be created during a local closure. However closure edges are simply oriented with the outer face on their right, hence may only create counterclockwise circuits.

This property is indeed quite strong: the following theorem ensures that the property of having no clockwise circuit characterizes the closure-tri-orientation and that a tri-orientation without clockwise circuit exists for any irreducible dissection. The proof of this theorem is delayed to Section 8.

THEOREM 4.4. Any irreducible dissection has a unique tri-orientation without clockwise circuit.

Recovering the tree: the opening mapping. Lemma 4.2 and the present section give all necessary elements to describe the inverse mapping of the closure, which is called the opening: let $D$ be an irreducible dissection endowed with its (unique by Theorem 4.4) tri-orientation without clockwise circuit. The opening of $D$ is the binary tree obtained from $D$ by deleting outer vertices, outer edges, and all inward half-edges.

\subsection{The closure is a bijection}

In this section, we show that the opening is inverse to the closure. By construction of the opening, the following lemma is straightforward:

LemMa 4.5. Let D be an irreducible dissection obtained as the closure of a binary tree $T$. Then the opening of $D$ is $T$. 
Conversely, the following also holds:

LEMMA 4.6. Let $T$ be a binary tree obtained as the opening of an irreducible dissection $D$. Then the closure of $T$ is $D$.

Proof. The proof relies on the definition of an order for removing inward halfedges. Start with the half-edges incident to outer vertices (that are all oriented inward): this clearly inverses the completion step of the closure. Each further removal must correspond to a local closure, that is, the removed half-edge must have the outer face on its right.

Let $M_{k}$ be the submap of the dissection induced by remaining half-edges after $k$ removals. Then $M_{k}$ covers the $n$ inner vertices, and, as long as some inward half-edge remains, it has at least $n$ entire edges (see Lemma 4.2). Hence, there is at least one cycle, and a simple one $\mathcal{C}$ can be extracted from the boundary of the outer face of $M_{k}$. Since there is no clockwise circuit, at least one edge of $\mathcal{C}$ is simply oriented with the interior of $\mathcal{C}$ on its left; the corresponding inward half-edge can be selected for the next removal.

Assuming Theorem 4.4, the bijective result follows from Lemmas 4.5 and 4.6:

TheOREM 4.7. For each $n \geq 1$, the closure mapping is a bijection between the set $\mathcal{B}_{n}$ of binary trees with $n$ nodes and the set $\mathcal{D}_{n}$ of irreducible dissections with $n$ inner vertices.

For each integer pair $(i, j)$ with $i+j \geq 1$, the closure mapping is a bijection between the set $\mathcal{B}_{i j}$ of bicolored binary trees with $i$ black nodes and $j$ white nodes, and the set $\mathcal{D}_{i j}$ of bicolored irreducible dissections with $i$ black inner vertices and $j$ white inner vertices.

The inverse mapping of the closure is the opening.

We can state three analogous versions of Theorem 4.7 for rooted objects:

THEOREM 4.8. The closure mapping induces the following correspondences between sets of rooted objects:

$$
\begin{aligned}
\mathcal{B}_{n}^{\prime} \times\{1, \ldots, 6\} & \equiv \mathcal{D}_{n}^{\prime} \times\{1, \ldots, n+2\}, \\
\mathcal{B}_{i j}^{\prime} \times\{1,2,3\} & \equiv \mathcal{D}_{i j}^{\prime} \times\{1, \ldots, i+j+2\}, \\
\mathcal{B}_{i j}^{\circ} \times\{1,2,3\} & \equiv \mathcal{D}_{i j}^{\prime} \times\{1, \ldots, 2 i-j+1\} .
\end{aligned}
$$

Proof. We define a bi-rooted irreducible dissection as a rooted irreducible dissection endowed with its tri-orientation without clockwise circuit and where a simply oriented edge is marked. We write $\mathcal{D}_{n}^{\prime \prime}$ for the set of bi-rooted irreducible dissections with $n$ inner vertices. Opening and rerooting on the stem corresponding to the marked edge defines a surjection from $\mathcal{D}_{n}^{\prime \prime}$ onto $\mathcal{B}_{n}^{\prime}$, for which each element of $\mathcal{B}_{n}^{\prime}$ has clearly six preimages, since the dissection could have been rooted at any edge of the hexagon. Moreover, erasing the mark clearly defines a surjection from $\mathcal{D}_{n}^{\prime \prime}$ to $\mathcal{D}_{n}^{\prime}$, for which each element of $\mathcal{D}_{n}^{\prime}$ has $n+2$ preimages according to Lemma 4.2. Hence, the closure defines a $(n+2)$-to- 6 mapping between $\mathcal{B}_{n}^{\prime}$ and $\mathcal{D}_{n}^{\prime}$. The proof of the $(i+j+2)$-to- 3 correspondence between $\mathcal{B}_{i j}^{\prime}$ and $\mathcal{D}_{i j}^{\prime}$ is the same.

The $(2 i-j+1)$-to- 3 correspondence between $\mathcal{B}_{i j}^{\bullet}$ and $\mathcal{D}_{i j}^{\prime}$ induced by the closure can be proved similarly, with the difference that the marked simply oriented edge 
has to have a black vertex as origin. Then the result follows from the fact that an object of $\mathcal{D}_{i j}^{\prime}$ endowed with its tri-orientation without clockwise circuit has $(2 i-j+1)$ simply oriented edges whose origin is a black vertex.

Let us mention that the $(i+j+2)$-to-3 correspondence between $\mathcal{B}_{i j}^{\prime}$ and $\mathcal{D}_{i j}^{\prime}$ is a key ingredient to the planar graph generators presented in [Fusy 2005].

The coefficient $\left|\mathcal{B}_{n}^{\prime}\right|$ is well-known to be the $n$-th Catalan number $\frac{1}{n+1}\left(\begin{array}{c}2 n \\ n\end{array}\right)$, and refinements of the standard proofs yield $\left|\mathcal{B}_{i j}^{\bullet}\right|=\frac{1}{2 j+1}\left(\begin{array}{c}2 j+1 \\ i\end{array}\right)\left(\begin{array}{c}2 i \\ j\end{array}\right)$, as detailed below in Section 4.5. Theorem 4.8 thus implies the following enumerative results:

COROLLARY 4.9. The coefficients counting rooted irreducible dissections have the following expressions,

$$
\begin{gathered}
\left|\mathcal{D}_{n}^{\prime}\right|=\frac{6}{n+2}\left|\mathcal{B}_{n}^{\prime}\right|=\frac{6}{(n+2)(n+1)}\left(\begin{array}{c}
2 n \\
n
\end{array}\right), \\
\left|\mathcal{D}_{i j}^{\prime}\right|=\frac{3}{2 i-j+1}\left|\mathcal{B}_{i j}^{\bullet}\right|=\frac{3}{(2 i+1)(2 j+1)}\left(\begin{array}{c}
2 j+1 \\
i
\end{array}\right)\left(\begin{array}{c}
2 i+1 \\
j
\end{array}\right) .
\end{gathered}
$$

These enumerative results have already been obtained by Mullin and Schellenberg [1968] using algebraic methods. Our method provides a direct bijective proof.

Notice that the cardinality of $\mathcal{D}_{n}^{\prime}$ is $\frac{1}{2} S(n, 2)$ where $S(n, m)=\frac{(2 n) !(2 m) !}{n ! m !(n+m) !}$ is the $n$-th super-Catalan number of order $m$. (These numbers are discussed by Gessel [1992].) Our bijection gives an interpretation of these numbers for $m=2$.

\subsection{Specialization to triangulations}

A nice feature of the closure mapping is that it specializes to a bijection between plane triangulations and a simple subfamily of binary trees. In this way, we get the first bijective proof for the formula giving the number of unrooted plane triangulations with $n$ vertices, found by Brown [1964], and recover the counting formula for rooted triangulations, already obtained by Tutte [1962] and by Poulalhon and Schaeffer [2006] using a different bijection.

THEOREM 4.10. The closure mapping is a bijection between the set $\mathcal{T}_{n}$ of (unrooted) plane triangulations with $n$ inner vertices and the set $\mathcal{S}_{n}$ of bicolored binary trees with $n$ black nodes and no stem (i.e., leaf) incident to a black node.

The closure mapping induces the following correspondence between the set $\mathcal{T}_{n}^{\prime}$ of rooted triangulations with $n$ inner vertices and the set $\mathcal{S}_{n}^{\prime}$ of trees in $\mathcal{S}_{n}$ rooted at a stem:

$$
\mathcal{S}_{n}^{\prime} \times\{1,2,3\} \equiv \mathcal{T}_{n}^{\prime} \times\{1, \ldots, 3 n+3\} .
$$

Proof. Plane triangulations are exactly 3 -connected planar maps where all faces have degree 3 . Hence, the angular mapping with border (Theorem 3.2) induces a bijection between $\mathcal{T}_{n}$ and the set of complete bicolored irreducible dissections with $n$ inner black vertices and all inner white vertices of degree 3 . In a tri-orientation, the indegree of each inner white vertex $v$ is $\operatorname{deg}(v)-3$ and the indegree of each outer white vertex $v$ is $\operatorname{deg}(v)-2$, hence the dissections considered here have no ingoing half-edge incident to a white vertex. Hence the opening of the dissection (by removing ingoing half-edges) is a binary tree with no stem incident to a black 


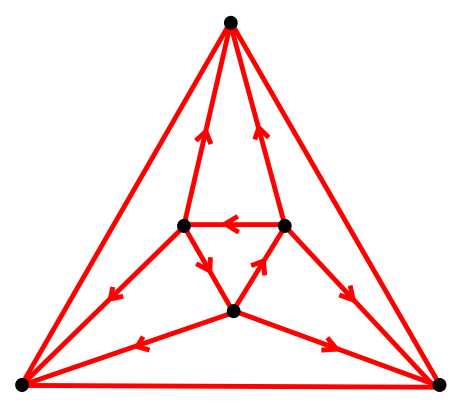

(a)

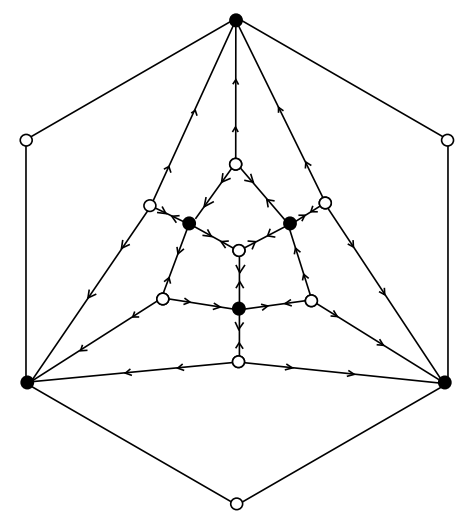

(c)

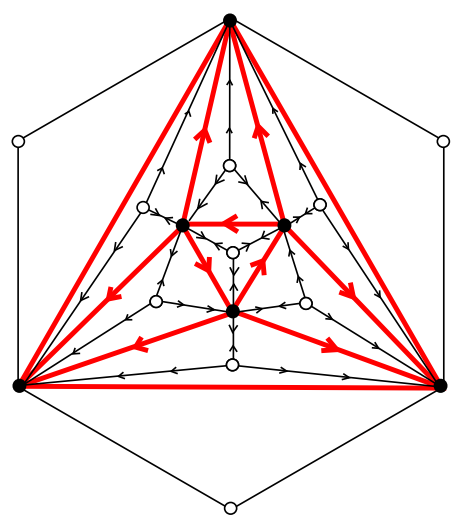

(b)

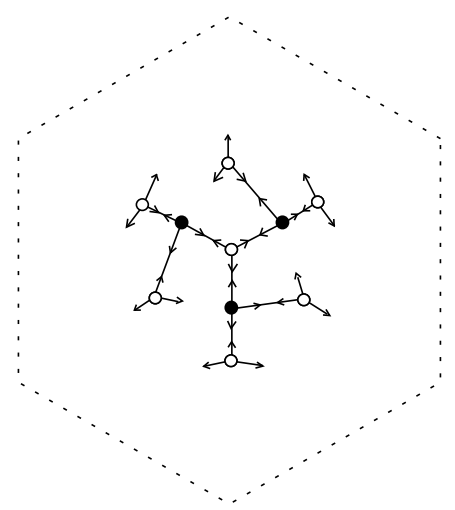

(d)

Fig. 7. The bijection between triangulations and bicolored binary trees with no leaf incident to a black node.

node. Conversely, starting from such a binary tree, the half-edges created during the closure mapping are opposite to a stem. As all stems are incident to white vertices, the half-edges created are incident to black vertices. Hence the degree of each white vertex does not increase during the closure mapping, i.e., remains equal to 3 for inner white vertices and equal to 2 for outer white vertices. This concludes the proof of the bijection $\mathcal{S}_{n} \equiv \mathcal{T}_{n}$.

The bijection $\mathcal{S}_{n}^{\prime} \times\{1,2,3\} \equiv \mathcal{T}_{n}^{\prime} \times\{1, \ldots, 3 n+3\}$ follows easily (see the proof of Theorem 4.8), using the fact that a tree of $\mathcal{S}_{n}$ has $3 n+3$ leaves.

This bijection, illustrated in Figure 7, makes it possible to count plane unrooted and rooted triangulations, as the subfamily of binary trees involved is easily enumerated.

Corollary 4.11. For $n \geq 0$, the number of rooted triangulations with $n$ inner ACM Journal Name, Vol. V, No. N, Month 20YY. 
vertices is

$$
\left|\mathcal{T}_{n}^{\prime}\right|=2 \frac{(4 n+1) !}{(n+1) !(3 n+2) !} .
$$

The number of unrooted plane triangulations with $n$ inner vertices is

$$
\begin{array}{rlrl}
\left|\mathcal{T}_{n}\right| & =\frac{2}{3} \frac{(4 n+1) !}{(n+1) !(3 n+2) !} & \text { if } n \equiv 2 \bmod 3, \\
\left|\mathcal{T}_{n}\right|=\frac{2}{3} \frac{(4 n+1) !}{(n+1) !(3 n+2) !}+\frac{4}{3} \frac{(4 k+1) !}{k !(3 k+2) !} & \text { if } n \equiv 1 \bmod 3[n=3 k+1], \\
\left|\mathcal{T}_{n}\right|=\frac{2}{3} \frac{(4 n+1) !}{(n+1) !(3 n+2) !}+\frac{2}{3} \frac{(4 k) !}{k !(3 k+1) !} & \text { if } n \equiv 0 \bmod 3[n=3 k] .
\end{array}
$$

ProOF. Let $\mathcal{S}^{\prime}=\cup_{n} \mathcal{S}_{n}^{\prime}$ be the class of rooted binary trees with no leaf incident to a black node and let $\mathcal{R}^{\prime}=\cup_{n} \mathcal{R}_{n}^{\prime}$ be the class of rooted binary trees where the root leaf is incident to a black node and all other leaves are incident to white nodes. Let $S(x)$ and $R(x)$ be the generating functions of $\mathcal{S}^{\prime}$ and $\mathcal{R}^{\prime}$ with respect to the number of black nodes. Clearly the two subtrees pending from the (white) root node of a tree of $\mathcal{S}^{\prime}$ are either empty or in $\mathcal{R}^{\prime}$. Hence $S(x)=(1+R(x))^{2}$. Similarly, a tree in $\mathcal{R}^{\prime}$ decomposes at the root node into two trees in $\mathcal{S}^{\prime}$, so that $R(x)=x S(x)^{2}$. Hence, $R(x)=x(1+R(x))^{4}$ is equal to the generating function of quaternary trees, and $S(x)=(1+R(x))^{2}$ is equal to the generating function of pairs of quaternary trees (the empty tree being allowed). Using a Lukaciewicz encoding and the cyclic lemma, the number of pairs of quaternary trees with a total of $n$ nodes is easily shown to be $\frac{2}{4 n+2} \frac{(4 n+2) !}{n !(3 n+2) !}$. This expression of $\left|\mathcal{S}_{n}^{\prime}\right|$ and the $(3 n+3)$-to- 3 correspondence between $\mathcal{S}_{n}^{\prime}$ and $\mathcal{T}_{n}^{\prime}$ yield the expression of $\left|\mathcal{T}_{n}^{\prime}\right|$.

Let us now prove the formula for $\left|\mathcal{T}_{n}\right|=\left|\mathcal{S}_{n}\right|$. Clearly, the only possible symmetry for a bicolored binary tree is a rotation of order 3 . Let $\mathcal{S}_{n}^{\text {sym }}$ be the set of trees of $\mathcal{S}_{n}$ with a rotation symmetry and let $\mathcal{S}_{n}^{\text {asy }}$ be the set of trees of $\mathcal{S}_{n}$ with no symmetry. Let $\mathcal{S}_{n}^{\prime \text { asy }}$ and $\mathcal{S}_{n}^{\prime \text { sym }}$ be the sets of trees of $\mathcal{S}_{n}^{\text {asy }}$ and $\mathcal{S}_{n}^{\text {sym }}$ that are rooted at a leaf. It is easily shown that a tree of $\mathcal{S}_{n}$ has $3 n+3$ leaves. Clearly the tree gives rise to $3 n+3$ rooted trees if it is asymmetric and gives rise to $n+1$ rooted trees if it is symmetric. Hence $\left|\mathcal{S}_{n}^{\text {asy }}\right|=\mid \mathcal{S}_{n}^{\prime}$ asy $\mid /(3 n+3)$ and $\left|\mathcal{S}_{n}^{\text {sym }}\right|=\mid \mathcal{S}_{n}^{\prime}$ sym $\mid /(n+1)$. Using $\left|\mathcal{S}_{n}\right|=\left|\mathcal{S}_{n}^{\text {asy }}\right|+\left|\mathcal{S}_{n}^{\text {sym }}\right|$ and $\left|\mathcal{S}_{n}^{\prime}\right|=\left|\mathcal{S}_{n}^{\text {asy }}\right|+\left|\mathcal{S}_{n}^{\prime \text { sym }}\right|$, we obtain

$$
\left|\mathcal{S}_{n}\right|=\frac{1}{3 n+3}\left|\mathcal{S}_{n}^{\prime}\right|+\frac{2}{3}\left|\mathcal{S}_{n}^{\mathrm{sym}}\right| .
$$

The centre of rotation of a tree in $\mathcal{S}_{n}^{\text {sym }}$ is either a black node, in which case $n=3 k+1$ for some integer $k \geq 0$, or is a white node, in which case $n=3 k$ for some integer $k \geq 0$. In the first case, a tree $\tau \in \mathcal{S}_{n}^{\text {sym }}$ is obtained by attaching to a black node 3 copies of a tree in $\mathcal{S}_{k}^{\prime}$. Hence $\left|\mathcal{S}_{3 k+1}^{\mathrm{sym}}\right|=\left|\mathcal{S}_{k}^{\prime}\right|=2 \frac{(4 k+1) !}{k !(3 k+2) !}$. In the second case, a tree $\tau \in \mathcal{S}_{n}^{\mathrm{sym}}$ is obtained by attaching to a white node 3 copies of a tree in $\mathcal{R}_{k}^{\prime}$. Hence $\left|\mathcal{S}_{3 k}^{\mathrm{sym}}\right|=\left|\mathcal{R}_{k}^{\prime}\right|=\frac{(4 k) !}{k !(3 k+1) !}$. The result follows.

\subsection{Counting, coding and sampling rooted bicolored binary trees}



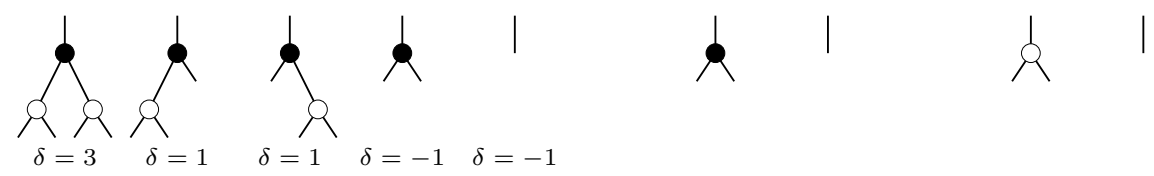

(a) $\mathcal{A}_{\bullet}$,

(b) $\mathcal{A}_{\bullet}$,

(c) $\mathcal{A}_{\circ}$.

Fig. 8. The three alphabets for words associated to bicolored binary trees.

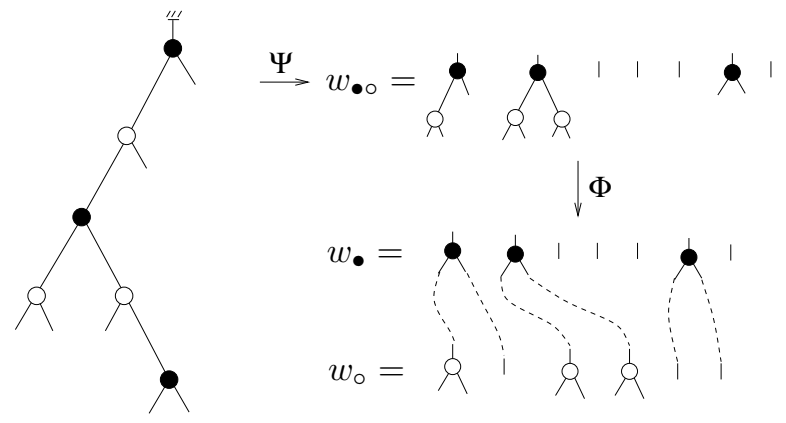

Fig. 9. A bicolored rooted binary tree, and the corresponding words $w_{\bullet}, w_{\bullet}$, and $w_{\circ}$.

4.5.1 From a bicolored tree to a pair of words. There exist general methods to encode a family of trees specified by several parameters. This section makes such methods explicit for the family of bicolored binary trees. Let $T$ be a black-rooted bicolored binary tree with $i$ black nodes and $j$ white nodes. Doing a depth-first traversal of $T$ from left to right, we obtain a word $w_{\bullet} \circ$ of length $(2 j+1)$ on the alphabet $\mathcal{A}_{\bullet}$ 。 represented in Figure 8(a), see Figure 9 for an example, the mapping being denoted by $\Psi$. Classically, the sum of the weights of the letters of any strict prefix of $w_{\bullet}$ is nonnegative and the sum of the weights of the letters of $w_{\bullet}$ is equal to -1 . In addition, $w_{\bullet}$ is the unique word in its cyclic equivalence-class that has these two properties.

The second step is to map $w_{\bullet \circ}$ to a pair $\left(w_{\bullet}, w_{\circ}\right):=\Phi\left(w_{\bullet \circ}\right)$ of words such that:

- $w_{\bullet}$ is a word of length $(2 j+1)$ on the alphabet $\mathcal{A}_{\bullet}$ shown in Figure $8(\mathrm{~b})$ with $i$ black-node-letters.

- $w_{\circ}$ is a word of length $2 i$ on the alphabet $\mathcal{A}_{\circ}$ shown in Figure 8(c) with $j$ white-node-letters.

Figure 9 illustrates the mapping $\Phi$ on an example.

4.5.2 Inverse mapping: from a pair of words to a tree. Conversely, let $\left(w_{\bullet}, w_{\circ}\right)$ be a pair of words such that $w_{\bullet}$ is of length $(2 j+1)$ on $\mathcal{A}_{\bullet}$ and has $i$ black-nodeletters, and $w_{\circ}$ is of length $2 i$ on $\mathcal{A}_{\circ}$ and has $j$ white-node-letters. First, to the pair $\left(w_{\bullet}, w_{\circ}\right)$ we associate a word $\widetilde{w}_{\bullet}$ of length $(2 j+1)$ on $\mathcal{A}_{\bullet}$ by doing the inverse of the mapping $\Phi$ shown in the right part of Figure 9 . The word $\widetilde{w}_{\bullet} \circ$ has the property that the sum of the weights of its letters is equal to -1 . There is a unique word $w_{\bullet} \circ$ in the cyclic equivalence-class of $\widetilde{w}_{\bullet}$ such that the sum of the weights of the letters of any strict prefix is nonnegative. We associate to $w_{\bullet}$ the binary tree of ACM Journal Name, Vol. V, No. N, Month 20YY. 
$\mathcal{B}_{i j}^{\bullet}$ obtained by doing the inverse of the mapping $\Psi$ shown in Figure 9.

This method allows us to sample uniformly objects of $\mathcal{B}_{i j}^{\bullet}$ in linear time and ensures that

$$
\left|\mathcal{B}_{i j}^{\bullet}\right|=\frac{1}{2 j+1}\left(\begin{array}{c}
2 j+1 \\
i
\end{array}\right)\left(\begin{array}{c}
2 i \\
j
\end{array}\right)
$$

\section{APPLICATION: COUNTING ROOTED 3-CONNECTED MAPS}

\subsection{Generating functions of rooted dissections}

Even if the counting formulas obtained in Corollary 4.9 are simple, it proves useful to have an expression of the corresponding generating functions. Indeed, the decomposition-method we develop is suitably handled by generating functions.

Let $r_{1}\left(x_{\bullet}, x_{\circ}\right):=\sum\left|\mathcal{B}_{i j}^{\bullet}\right| x_{\bullet}^{i} x_{\circ}^{j}$ and $r_{2}\left(x_{\bullet}, x_{\circ}\right):=\sum\left|\mathcal{B}_{i j}^{\circ}\right| x_{\bullet}^{i} x_{\circ}^{j}$ be the series of black-rooted and white-rooted bicolored binary trees. By decomposition at the root, $r_{1}\left(x_{\bullet}, x_{\circ}\right)$ and $r_{2}\left(x_{\bullet}, x_{\circ}\right)$ are the solutions of the system:

$$
\left\{\begin{array}{l}
r_{1}\left(x_{\bullet}, x_{\circ}\right)=x_{\bullet}\left(1+r_{2}\left(x_{\bullet}, x_{\circ}\right)\right)^{2}, \\
r_{2}\left(x_{\bullet}, x_{\circ}\right)=x_{\circ}\left(1+r_{1}\left(x_{\bullet}, x_{\circ}\right)\right)^{2} .
\end{array}\right.
$$

Define an edge-marked bicolored binary tree as a bicolored binary tree with a marked inner edge. Let $\overline{\mathcal{B}}_{i j}$ be the set of edge-marked bicolored binary trees with $i$ black nodes and $j$ white nodes. Cutting the marked edge of such a tree yields a pair made of a black-rooted and a white-rooted binary tree. As a consequence, the generating function counting edge-marked bicolored binary trees is $r_{1} \cdot r_{2}$, i.e., $r_{1} \cdot r_{2}=\sum_{i j}\left|\overline{\mathcal{B}}_{i j}\right| x_{\bullet}^{i} x_{\circ}^{j}$.

Let us consider bi-rooted objects as in the proof of Theorem 4.8; since any object of $\mathcal{B}_{i j}$ has $(2 i-j+1)$ white leaves (connected to a black node) and $(2 j-i+1)$ black leaves (connected to a white node),

$$
\left|\mathcal{B}_{i j}^{\circ}\right|=\frac{2 j-i+1}{2 i-j+1}\left|\mathcal{B}_{i j}^{\bullet}\right| .
$$

Similarly, counting in two ways the objects of $\mathcal{B}_{i j}^{\bullet}$ having a marked edge yields

$$
\left|\overline{\mathcal{B}}_{i j}\right|=\frac{i+j-1}{2 i-j+1}\left|\mathcal{B}_{i j}^{\bullet}\right| .
$$

Thus, we have $\left|\mathcal{B}_{i j}^{\bullet}\right|+\left|\mathcal{B}_{i j}^{\circ}\right|-\left|\overline{\mathcal{B}}_{i j}\right|=\frac{3}{2 i-j+1}\left|\mathcal{B}_{i j}^{\bullet}\right|=\left|\mathcal{D}_{i j}^{\prime}\right|$ (using (3)), so that

$$
\sum_{i, j}\left|\mathcal{D}_{i j}^{\prime}\right| x_{\bullet}^{i} x_{\circ}^{j}=r_{1}\left(x_{\bullet}, x_{\circ}\right)+r_{2}\left(x_{\bullet}, x_{\circ}\right)-r_{1}\left(x_{\bullet}, x_{\circ}\right) r_{2}\left(x_{\bullet}, x_{\circ}\right) .
$$

Substituting $x_{\bullet}$ and $x_{\circ}$ by $x$, we obtain:

$$
\sum_{n}\left|\mathcal{D}_{n}^{\prime}\right| x^{n}=2 r(x)-r(x)^{2},
$$

where $r(x)=x(1+r(x))^{2}$ is the generating function of binary trees according to the number of inner nodes. 


\subsection{Generating function of rooted 3-connected maps}

Injection from $\mathcal{Q}^{\prime}$ to $\mathcal{D}^{\prime}$. Let us consider the mapping $\iota$ defined on rooted quadrangulations by the removal of the root-edge and rerooting on the next edge in counterclockwise order around the root-vertex; $\iota$ is clearly injective, and for any quadrangulation $Q, \iota(Q)$ has only quadrangular faces but the outer one, which is hexagonal. In addition, $\iota(Q)$ can not have more separating 4-cycles than $Q$. Hence the restriction of $\iota$ to $\mathcal{Q}^{\prime}$ is an injection from $\mathcal{Q}^{\prime}$ to $\mathcal{D}^{\prime}$, more precisely from $\mathcal{Q}_{n}^{\prime}$ to $\mathcal{D}_{n-4}^{\prime}$ and from $\mathcal{Q}_{i j}^{\prime}$ to $\mathcal{D}_{i-3, j-3}^{\prime}$.

It is however not a bijection, since the inverse edge-adding operation $\pi$, performed on an irreducible dissection, can create a separating 4-cycle on the obtained quadrangulation. Precisely, given $D$ a rooted irreducible dissection - with $s$ the root-vertex and $t$ the vertex of the hexagon opposite to $s$ - a path of length 3 between $s$ and $t$ is called a decomposition path. The two paths of edges of the hexagon connecting $s$ to $t$ are called outer decomposition paths, and the other ones, if any, are called inner decomposition paths of $D$.

Observe that inner decomposition paths of $D$ are in one-to-one correspondence with separating 4-cycles of the quadrangulation $\pi(D)$ (i.e., the quadrangulation obtained from $D$ by adding a root-edge between $s$ and $t$ oriented out of $s$ ).

A rooted irreducible dissection without inner decomposition path is said to be undecomposable. The corresponding class is denoted by $\mathcal{U}^{\prime}$. The discussion on decomposition paths yields the following result.

Lemma 5.1. Denote by $\mathcal{U}_{n}^{\prime}$ the set of rooted undecomposable dissections with $n$ inner vertices and by $\mathcal{U}_{i j}^{\prime}$ the set of rooted undecomposable dissections with $i$ inner black vertices and $j$ inner white vertices. Then $\mathcal{U}_{n-4}^{\prime}$ is in bijection with $\mathcal{P}_{n}^{\prime}$ and $\mathcal{U}_{i-3, j-3}^{\prime}$ is in bijection with $\mathcal{P}_{i j}^{\prime}$.

Proof. A rooted irreducible quadrangulation is mapped by $\iota$ to a rooted dissection such that the inverse edge-adding operation $\pi$ does not create a separating 4-cycle, i.e., an undecomposable dissection. Moreover, Euler's relation ensures that the image of a quadrangulation with $n$ faces has $n-4$ inner vertices. By injectivity, $\iota$ is bijective to its image, i.e., $\iota$ is a bijection between $\mathcal{Q}_{n}^{\prime}$ and $\mathcal{U}_{n-4}^{\prime}$; and a bijection between $\mathcal{Q}_{i j}^{\prime}$ and $\mathcal{U}_{i-3, j-3}^{\prime}$. The result follows, as $\mathcal{Q}_{n}^{\prime}$ and $\mathcal{Q}_{i j}^{\prime}$ are respectively in bijection with $\mathcal{P}_{n}^{\prime}$ and $\mathcal{P}_{i j}^{\prime}$ via the angular mapping (Theorem 3.1).

Thanks to Lemma 5.1, enumerating rooted 3-connected maps reduces to enumerating rooted undecomposable dissections.

Decomposition of rooted irreducible dissections. Since irreducible dissections do not have multiple edges nor cycles of odd length, decomposition paths satisfy the following properties:

Lemma 5.2. Let $D$ be a rooted irreducible dissection, and let $\mathcal{P}_{1}$ and $\mathcal{P}_{2}$ be two different decomposition paths of $D$. Then:

- either $\mathcal{P}_{1} \cap \mathcal{P}_{2}=\{s, t\}$, in which case $\mathcal{P}_{1}$ and $\mathcal{P}_{2}$ are said to be internally disjoint;

- or there exists one inner vertex $v$ such that $\mathcal{P}_{1} \cap \mathcal{P}_{2}=\{s\} \cup\{t\} \cup\{v\}$, in which case $\mathcal{P}_{1}$ and $\mathcal{P}_{2}$ are said to be upper or lower joint whether $v$ is adjacent to $s$ or $t$.

ACM Journal Name, Vol. V, No. N, Month 20YY. 


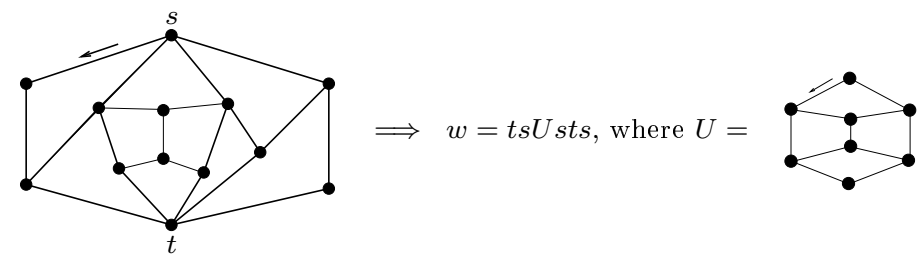

Fig. 10. Example of decomposition of a rooted irreducible dissection and of its associated decomposition word.

Lemma 5.2 implies in particular that two decomposition paths can not cross each other. Hence the decomposition paths of an irreducible dissection $D$ follow a leftto-right order, from the outer decomposition path containing the root — called left outer path - to the other outer decomposition path - called right outer path.

Lemma 5.3. Let $D$ be a rooted irreducible dissection, and let $\mathcal{P}_{1}$ and $\mathcal{P}_{2}$ be two upper joint (resp. lower joint) decomposition paths of $D$. Then the interior of the area delimited by $\mathcal{P}_{1}$ and $\mathcal{P}_{2}$ consists of a unique face incident to $t$ (resp. to $s$ ).

Proof. Follows from the fact that the interior of each 4-cycle of $D$ is a face.

Decomposition word of an irreducible dissection. Let $D \in \mathcal{D}^{\prime}$ and let $\left\{\mathcal{P}_{0}, \ldots, \mathcal{P}_{\ell}\right\}$ be the sequence of decomposition paths of $D$ ordered from left to right. Let us consider the alphabet $\mathcal{A}=\{s\} \cup\{t\} \cup \mathcal{U}^{\prime}$; the decomposition word of $D$ is the word $w=w_{1} \ldots w_{\ell}$ of length $\ell$ on $\mathcal{A}$ such that, for any $1 \leq i \leq \ell$ : if $\mathcal{P}_{i-1}$ and $\mathcal{P}_{i}$ are upper joint, then $w_{i}=s$; if $\mathcal{P}_{i-1}$ and $\mathcal{P}_{i}$ are lower joint, then $w_{i}=t$; if $\mathcal{P}_{i-1}$ and $\mathcal{P}_{i}$ are internally disjoint, then $w_{i}=U$, where $U$ is the undecomposable dissection delimited by $\mathcal{P}_{i-1}$ and $\mathcal{P}_{i}$, rooted at the first edge of $\mathcal{P}_{i-1}$ and with $s$ as root-vertex, see Figure 10. This encoding is injective, an easy consequence of Lemma 5.3.

Characterization of decomposition words of elements of $\mathcal{D}^{\prime}$. The fact that $D$ has no separating 4-cycle easily implies that its decomposition word has no factor ss nor $t t$, and these are the only forbidden factors. Moreover, as a dissection has at least one inner vertex, a decomposition word can neither be the empty word, nor the one-letter words $s$ and $t$, nor the two-letter words $s t$ and $t s$. It is easily seen that all other words encode irreducible dissections of the hexagon.

This leads to the following equation linking the generating functions $D(x)$ and $U(x)$ counting $\mathcal{D}^{\prime}$ and $\mathcal{U}^{\prime}$ according to the number of inner vertices,

$$
x^{2} D(x)+2 x^{2}+2 x+1=\left(1+\frac{2 x}{1-x}\right) \cdot \frac{1}{1-x^{2} U(x)\left(1+\frac{2 x}{1-x}\right)} .
$$

Similarly, let $D\left(x_{\bullet}, x_{\circ}\right):=\sum\left|\mathcal{D}_{i j}^{\prime}\right| x_{\bullet}^{i} x_{\circ}^{j}$ and $U\left(x_{\bullet}, x_{\circ}\right):=\sum\left|\mathcal{U}_{i j}^{\prime}\right| x_{\bullet}^{i} x_{\circ}^{j}$. Then the characterization of the coding words gives

$$
\begin{aligned}
& x_{\bullet} x_{\circ} D\left(x_{\bullet}, x_{\circ}\right)+2 x_{\bullet} x_{\circ}+x_{\bullet}+x_{\circ}+1 \\
= & \left(1+x_{\bullet}\right) \cdot \frac{1}{1-x_{\circ} x_{\bullet}} \cdot\left(1+x_{\circ}\right) \cdot \frac{1}{1-x_{\bullet} x_{\circ} U\left(x_{\bullet}, x_{\circ}\right)\left(1+x_{\bullet}\right) \frac{1}{1-x_{\circ} x_{\bullet}}\left(1+x_{\circ}\right)} .
\end{aligned}
$$


THEOREM 5.4. Let $\mathcal{P}_{n}^{\prime}$ be the number of rooted 3-connected maps with $n$ edges and $\mathcal{P}_{i j}^{\prime}$ the number of rooted 3-connected maps with $i$ vertices and $j$ faces. Then

$$
\sum_{n}\left|\mathcal{P}_{n+2}^{\prime}\right| x^{n}=\frac{1-x}{1+x}-\frac{1}{1+2 x+2 x^{2}+x^{2}\left(2 r(x)-r(x)^{2}\right)},
$$

where $r(x)=x(1+r(x))^{2}$, and

$$
\begin{aligned}
\sum_{i, j}\left|\mathcal{P}_{i+2, j+2}^{\prime}\right| x_{\bullet}^{i} x_{\circ}^{j} \\
\quad=\frac{1-x_{\bullet} x_{\circ}}{\left(1+x_{\bullet}\right)\left(1+x_{\circ}\right)}-\frac{1}{1+x_{\bullet}+x_{\circ}+2 x_{\bullet} x_{\circ}+x_{\bullet} x_{\circ}\left(r_{1}+r_{2}-r_{1} r_{2}\right)},
\end{aligned}
$$

where $\left\{\begin{array}{l}r_{1}\left(x_{\bullet}, x_{\circ}\right)=x_{\bullet}\left(1+r_{2}\left(x_{\bullet}, x_{\circ}\right)\right)^{2} \\ r_{2}\left(x_{\bullet}, x_{\circ}\right)=x_{\circ}\left(1+r_{1}\left(x_{\bullet}, x_{\circ}\right)\right)^{2}\end{array}\right.$.

Proof. Lemma 5.1 ensures that $\sum_{n}\left|\mathcal{P}_{n+2}^{\prime}\right| x^{n}=x^{2} U(x)$ and, more precisely, $\sum_{i, j}\left|\mathcal{P}_{i+2, j+2}^{\prime}\right| x_{\bullet}^{i} x_{\circ}^{j}=x_{\bullet} x_{\circ} U\left(x_{\bullet}, x_{\circ}\right)$. Moreover, Equations (8) and Equation (9) yield expressions of $x^{2} U(x)$ and $x_{\bullet} x_{\circ} U\left(x_{\bullet}, x_{\circ}\right)$ respectively in terms of $D(x)$ and $D\left(x_{\bullet}, x_{\circ}\right)$. In these expressions, replace $D(x)$ and $D\left(x_{\bullet}, x_{\circ}\right)$ by their respective expression in terms of $r$ and of $r_{1}$ and $r_{2}$, as given by Equations (6) and (7).

\section{APPLICATION: SAMPLING ROOTED 3-CONNECTED MAPS}

\subsection{Sampling rooted 3-connected maps with $n$ edges}

Theorem 4.8 (first identity) ensures that the following algorithm samples rooted 3 -connected maps with $n$ edges uniformly at random:

(1) Sample an object $T \in \mathcal{B}_{n-4}^{\prime}$ uniformly (e.g. using parenthesis words).

(2) Perform the closure of $T$ to obtain an irreducible dissection $D$ with $(n-4)$ vertices. Choose randomly one of the six edges of the hexagon of $D$ to carry the root. If $D$ is not undecomposable, then reject and restart.

(3) Connect by a new edge $e$ the root-vertex of $D$ to the opposite outer vertex. Take $e$ as root edge, with the same root-vertex as in $D$. This gives a rooted irreducible quadrangulation $Q$ with $n$ faces.

(4) Return the rooted 3-connected map in $\mathcal{P}_{n}^{\prime}$ associated to $Q$ by the angular mapping.

Proposition 6.1. The success probability of the sampler at each trial is equal to $\left|\mathcal{P}_{n}^{\prime}\right| /\left|\mathcal{D}_{n-4}^{\prime}\right|$, which satisfies

$$
\frac{\left|\mathcal{P}_{n}^{\prime}\right|}{\left|\mathcal{D}_{n-4}^{\prime}\right|} \underset{n \rightarrow \infty}{\rightarrow} \frac{2^{8}}{3^{6}}
$$

Hence, the number of rejections follows a geometric law whose mean is asymptotically $c=3^{6} / 2^{8}$. As the closure mapping has linear-time complexity, the sampling algorithm has expected linear-time complexity.

Proof. According to Section 4.3, $\left|\mathcal{D}_{n}^{\prime}\right|=\frac{6}{n+2}\left|\mathcal{B}_{n}^{\prime}\right|=\frac{6(2 n) !}{(n+2) ! n !}$. Stirling formula yields $\left|\mathcal{D}_{n-4}^{\prime}\right| \sim \frac{3}{128 \sqrt{\pi}} \frac{4^{n}}{n^{5 / 2}}$. Moreover, according to [Tutte 1963], $\left|\mathcal{P}_{n}^{\prime}\right| \sim \frac{2}{3^{5} \sqrt{\pi}} \frac{4^{n}}{n^{5 / 2}}$. This yields the limit of $\left|\mathcal{P}_{n}^{\prime}\right| /\left|\mathcal{D}_{n-4}^{\prime}\right|$. 


\subsection{Sampling rooted 3-connected maps with $i$ vertices and $j$ faces}

Similarly, Theorem 4.8 (third identity), ensures that the following algorithm samples rooted 3 -connected maps with $i$ vertices and $j$ faces uniformly at random:

(1) Sample an object $T \in \mathcal{B}_{i-3, j-3}^{\bullet}$ uniformly at random. A simple method is described in Section 4.5.2.

(2) Perform the closure of $T$ to obtain an irreducible dissection $D$ with $(i-3)$ inner black vertices and $(j-3)$ inner white vertices. Choose randomly the root-vertex among the three black vertices of the hexagon. If the dissection is not undecomposable, then reject and restart.

(3) Connect by a new edge $e$ the root-vertex of $D$ to the opposite outer vertex. Take $e$ as root edge, with the same root-vertex as in $D$. This gives a rooted irreducible quadrangulation $Q$ with $i$ black vertices and $j$ white vertices.

(4) Return the rooted 3-connected map in $\mathcal{P}_{i j}^{\prime}$ associated to $Q$ by the angular mapping.

Proposition 6.2. The success probability of the sampler at each trial is equal to $\left|\mathcal{P}_{i j}^{\prime}\right| /\left|\mathcal{D}_{i-3, j-3}^{\prime}\right|$. Let $\left.\alpha \in\right] 1 / 2,2\left[\right.$; if $i$ and $j$ are correlated by $\frac{i}{j} \rightarrow \alpha$ as $i \rightarrow \infty$, then

$$
\frac{\left|\mathcal{P}_{i j}^{\prime}\right|}{\left|\mathcal{D}_{i-3, j-3}^{\prime}\right|} \sim \frac{2^{8}}{3^{6}} \frac{(2-\alpha)^{2}(2 \alpha-1)^{2}}{\alpha^{2}}=: \frac{1}{c_{\alpha}} .
$$

Hence, when $\frac{i}{j} \rightarrow \alpha$, the number of rejections follows a geometric law whose mean is asymptotically $c_{\alpha}$. Under these conditions, the sampling algorithm has an expected linear-time complexity, the linearity factor being asymptotically proportional to $c_{\alpha}$.

Moreover, in the worst case of triangulations where $j=2 i-4$, the mean number of rejections is quadratic, so that the sampling complexity is cubic.

Proof. These asymptotic results are easy consequences of the expression of $\left|\mathcal{D}_{i j}^{\prime}\right|$ obtained in Corollary 4.9 and of the asymptotic result $\left|\mathcal{P}_{i j}^{\prime}\right| \sim \frac{1}{3^{5} 2^{2} i j}\left(\begin{array}{c}2 i-2 \\ j+2\end{array}\right)\left(\begin{array}{c}2 j-2 \\ i+2\end{array}\right)$ given in [Bender 1987].

\section{APPLICATION: CODING 3-CONNECTED MAPS}

This section introduces an algorithm, derived from the inverse of the closure mapping, to encode a 3-connected map. Precisely, the algorithm encodes an outertriangular 3-connected map, but it is then easily extended to encode any 3 -connected map. Indeed, if the outer face of $G$ is not triangular, fix three consecutive vertices $v, v^{\prime}$ and $v^{\prime \prime}$ incident to the outer face of $G$ and link $v$ and $v^{\prime \prime}$ by an edge to obtain an outer-triangular 3 -connected planar map $\widetilde{G}$; the coding of $G$ is obtained as the coding of $\widetilde{G}$ plus one bit indicating if an edge-addition has been done.

\subsection{Description of the coding algorithm}

Let $G$ be an outer-triangular 3-connected map and let $G^{\prime}$ be its derived map, as defined in Section 3.2. The coding algorithm relies on the following steps, illustrated in Figure 11. 


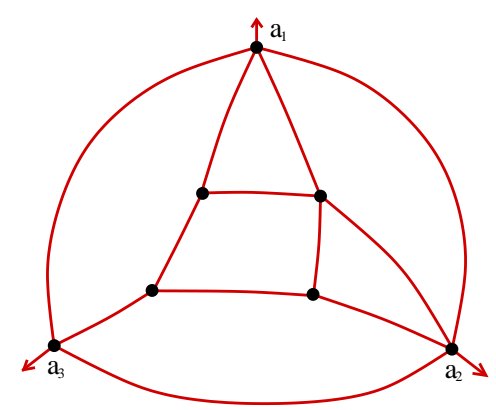

(a)

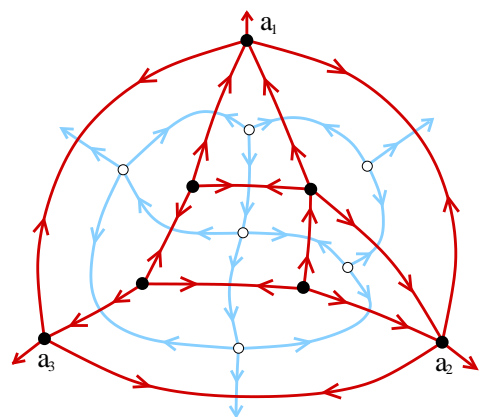

(c)

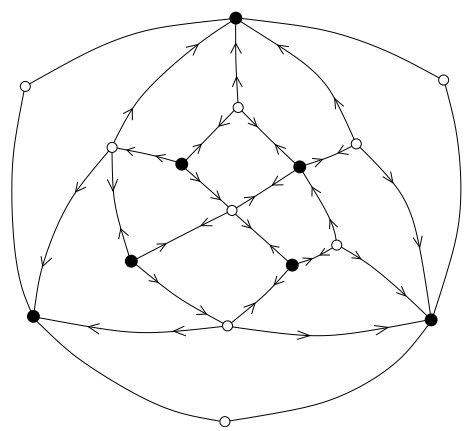

(e)

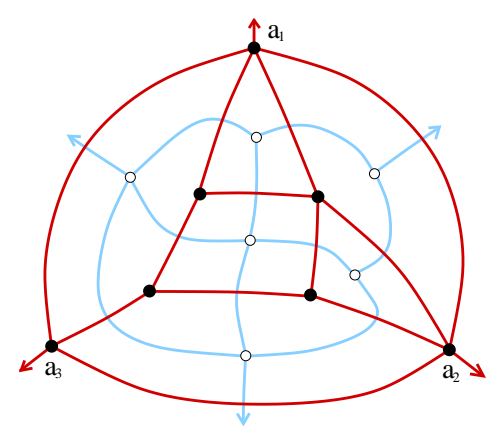

(b)

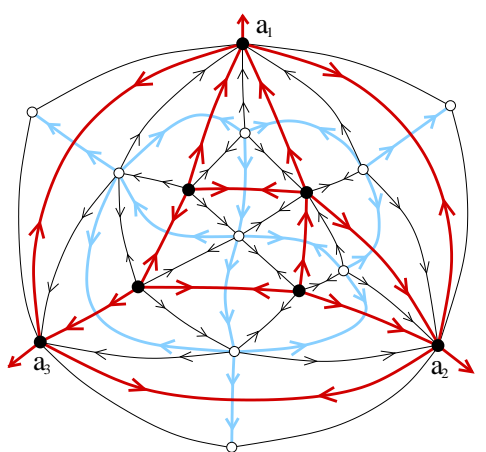

(d)

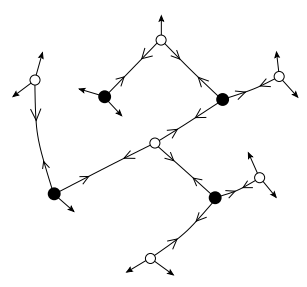

(f)

Fig. 11. Execution of the encoding algorithm on an example.

7.1.1 Compute a particular orientation of the derived map $G^{\prime}$ (Fig. 11(b)-(c)). The first step of the algorithm is to compute a specific orientation $X_{0}$ of the edges of the derived map $G^{\prime}$, such that $X_{0}$ has no clockwise circuit, each primal or dual vertex has outdegree 3 and each edge-vertex has outdegree 1 . Such an orientation of $G^{\prime}$ exists and is unique, as we will see in Theorem 8.1. A linear time algorithm to compute $X_{0}$ is given in Section 9.

7.1.2 Compute the irreducible dissection $D$ associated to $G$ (Fig. 11(d)). Consider the bicolored complete irreducible dissection $D$ associated to $G$ by the bijection ACM Journal Name, Vol. V, No. N, Month 20YY. 
presented in Section 3.2 (and reformulated in Section 3.3), i.e., the dissection having the same derived map as $G$. Notice that $D$ has $n$ inner faces if $G$ has $n$ edges. Hence, according to Euler's relation, $D$ has $n-2$ inner vertices. Similarly, if $G$ has $i$ vertices and $j$ inner faces, then $D$ has $i$ black vertices and $j+3$ white vertices.

7.1.3 Compute the tri-orientation of $D$ without clockwise circuit (Fig. 11(d)). We orient each half-edge $h$ of $D$ belonging to an inner edge as follows: $h$ is directed inward if its incident vertex belongs to the hexagon; otherwise, $h$ receives the orientation of the cw-following edge of $G^{\prime}$. As shown in Section 8 (more precisely in Lemma 8.13, composed with the correspondence of Figure 13), this process yields the unique tri-orientation of $D$ without clockwise circuit.

7.1.4 Open the dissection $D$ into a binary tree $T$ (Fig. 11(f)). Once the tri-orientation without clockwise circuit is computed, $D$ is opened into a binary tree $T$, by deleting outer vertices, outer edges, and all ingoing half-edges (see Section 4.2).

7.1.5 Encode the tree $T$. First, choose an arbitrary leaf of $T$, root $T$ at this leaf, and encode the obtained rooted binary tree using a parenthesis word (also called Dyck word). The opening of a 3 -connected map with $n$ edges is a binary tree with $n-2$ inner nodes, yielding an encoding Dyck word of length $2(n-2)$.

Similarly, the opening of a 3 -connected map with $i$ vertices and $j$ inner faces is a black-rooted bicolored binary tree with $i-3$ black nodes and $j$ white nodes. A black-rooted bicolored binary trees with a given number of black and white nodes is encoded by a pair of words, as explained in Section 4.5.1. Then the two words can be asymptotically optimally encoded in linear time, according to [Bonichon et al. 2003, Lem.7].

THEOREM 7.1. The coding algorithm has linear-time complexity and is asymptotically optimal: the number of bits per edge of the code of a map in $\mathcal{P}_{n}^{\prime}$ (resp. in $\left.\mathcal{P}_{i j}^{\prime}\right)$ is asymptotically equal to the binary entropy per edge, defined as $\frac{1}{n} \log _{2}\left(\left|\mathcal{P}_{n}^{\prime}\right|\right)$ (resp. $\frac{1}{i+j-2} \log _{2}\left(\left|\mathcal{P}_{i j}^{\prime}\right|\right)$ ).

Proof. It is clear that the encoding algorithm has linear-time complexity, provided the algorithm computing the constrained orientation without clockwise circuit of the derived map has linear-time complexity (which will be proved in Section 9 and Section 10).

According to Corollary 4.9, Proposition 6.1 and $6.2,\left|\mathcal{B}_{n}^{\prime}\right| /\left|\mathcal{P}_{n}^{\prime}\right|$ and $\left|\mathcal{B}_{i j}^{\bullet}\right| /\left|\mathcal{P}_{i j}^{\prime}\right|$ are bounded by fixed polynomials. Hence, the entropy per edge of $\mathcal{B}_{n}^{\prime}$ and $\mathcal{P}_{n}^{\prime}$ are asymptotically equal, and the binary entropy per edge of $\mathcal{B}_{i j}^{\bullet}$ and $\mathcal{P}_{i j}^{\prime}$ are asymptotically equal. As the encoding of objects of $\mathcal{B}_{n}^{\prime}\left(\mathcal{B}_{i j}^{\bullet}\right)$ using parenthesis words is asymptotically optimal, the encoding of objects of $\mathcal{P}_{n}^{\prime}\left(\mathcal{P}_{i j}^{\prime}\right.$, respectively) is also asymptotically optimal.

\section{PROOF OF THEOREM 4.4}

This section is devoted to the proof of Theorem 4.4, which states that each irreducible dissection has a unique tri-orientation without clockwise circuit. 


\section{1 $\alpha$-orientations and outline of the proof}

Definition. Let $G=(V, E)$ be a planar map. Consider a function $\alpha: V \rightarrow \mathbb{N}$. An $\alpha$-orientation of $G$ is an orientation of the edges of $G$ such that the outdegree of each vertex $v$ of $G$ is $\alpha(v)$. If an $\alpha$-orientation exists, then the function $\alpha$ is said to be feasible for $G$.

Existence and uniqueness of $\alpha$-orientations. The following results are proved in [Felsner 2004] (the first point had already been proved in [Ossona de Mendez 1994]):

TheOrEm 8.1 ([FELSNer 2004]). Given a planar map $G$ and a feasible function $\alpha$, there exists a unique $\alpha$-orientation of $G$ without clockwise circuit. This $\alpha$-orientation is called the minimal ${ }^{1} \alpha$-orientation of $G$.

Given the derived map of an outer-triangular 3-connected planar map, the function $\alpha_{0}$ such that $\alpha_{0}(v)=3$ for all primal and dual vertices and $\alpha_{0}(v)=1$ for all edge-vertices is a feasible function.

Theorem 8.1 ensures uniqueness of the orientation without clockwise circuit of a graph with prescribed outdegree for each vertex. However, this property does not directly imply uniqueness in Theorem 4.4, because a tri-orientation has bi-oriented edges.

To use Theorem 8.1, we work with the derived map $G^{\prime}$ of an irreducible dissection $D$, as defined in Section 3.3. We have defined derived maps only for a subset of irreducible dissections, namely for bicolored complete irreducible dissections (recall that these are bicolored dissections such that the 3 outer white vertices have degree 2). As a consequence, a first step toward proving Theorem 4.4 is to reduce its proof to the proof of existence and uniqueness of a so-called complete-tri-orientation (a slight adaptation of the definition of tri-orientation) without clockwise circuit for any bicolored complete irreducible dissection.

We prove that a complete-tri-orientation without clockwise circuit of a bicolored complete irreducible dissection $D$ is transposed injectively into an $\alpha_{0}$-orientation without clockwise circuit of its derived map $G^{\prime}$. By injectivity and by uniqueness of the $\alpha_{0}$-orientation without clockwise circuit of $G^{\prime}$, this implies uniqueness of a tri-orientation without clockwise circuit for $D$.

The final step will be to prove that an $\alpha_{0}$-orientation without clockwise circuit of $G^{\prime}$ is transposed into a complete-tri-orientation without clockwise circuit of $D$. By existence of an $\alpha_{0}$-orientation without clockwise circuit for $G^{\prime}$ (Theorem 8.1), this implies the existence of a complete-tri-orientation without clockwise circuit of $D$.

\subsection{Reduction to the case of bicolored complete dissections}

Introduction. The aim of this section is to reduce the proof of Theorem 4.4 to the class of complete bicolored irreducible dissections. We state the following proposition where the term "complete-tri-orientation", to be defined later, is a slight adaptation of the notion of tri-orientation.

Proposition 8.2. The existence and uniqueness of a complete-tri-orientation without clockwise circuit for any bicolored complete irreducible dissection implies

\footnotetext{
${ }^{1}$ The term minimal refers to the fact that the set of all $\alpha$-orientations of $G$ forms a distributive lattice, the "flip" operation being a circuit reversion.
}

ACM Journal Name, Vol. V, No. N, Month 20YY. 
the existence and uniqueness of a tri-orientation without clockwise circuit for any irreducible dissection, i.e., implies Theorem 4.4.

The rest of this subsection is devoted to the proof of Proposition 8.2. The proof is done in two steps. First, reduce the proof of Theorem 4.4 to the existence and uniqueness of a tri-orientation without clockwise circuit for any bicolored complete irreducible dissection. Then, prove that this reduces to the existence and uniqueness of a complete-tri-orientation without clockwise circuit for any bicolored complete irreducible dissection.

Completion of a bicolored irreducible dissection. For any bicolored irreducible dissection $D$, we define its completed dissection $D^{c}$ as follows. For each white vertex $v$ of the hexagon, we denote by $e_{\mathrm{l}}(v)\left(e_{\mathrm{r}}(v)\right)$ the outer edge starting from $v$ with the interior of the hexagon on the left (right, respectively) and denote by $l(v)$ and $r(v)$ the neighbours of $v$ incident to $e_{1}(v)$ and to $e_{\mathrm{r}}(v)$. We perform the following operation: if $v$ has degree at least 3 , a new white vertex $v^{\prime}$ is created outside of the hexagon and is linked to $l(v)$ and to $r(v)$ by two new edges $e_{\mathrm{l}}\left(v^{\prime}\right)$ and $e_{\mathrm{r}}\left(v^{\prime}\right)$, see Figure 12. The vertex $v^{\prime}$ is said to cover the vertex $v$.

The dissection obtained is a bicolored dissection of the hexagon such that the three white vertices of the hexagon have two incident edges, see the transition between Figure 13(a) and Figure 13(b) (ignore here the orientation of edges).

Lemma 8.3. The completion $D^{c}$ of a bicolored irreducible dissection $D$ is a bicolored complete irreducible dissection.

Proof. The outer white vertices of $D^{c}$ have degree 2 by construction. Hence, we just have to prove that $D^{c}$ is irreducible. As $D$ is irreducible, if a separating 4-cycle $\mathcal{C}$ appears in $D^{c}$ when the completion is performed, then it must contain a white vertex $v^{\prime}$ of the hexagon of $D^{c}$ added during the completion, so as to cover an outer white vertex $v$ of degree greater than 2. Two edges of $\mathcal{C}$ are the edges $e_{1}\left(v^{\prime}\right)$ and $e_{\mathrm{r}}\left(v^{\prime}\right)$ incident to $v^{\prime}$ in $D^{c}$. The two other edges $\epsilon_{1}$ and $\epsilon_{2}$ of $\mathcal{C}$ form a path of length 2 connecting the vertices $l(v)$ and $r(v)$ and passing by the interior of $D$ (otherwise, $\mathcal{C}$ would enclose a face). As $D$ is irreducible, the 4-cycle $\mathcal{C}^{\prime}$ of $D$ consisting of the edges $e_{1}(v), e_{\mathrm{r}}(v), \epsilon_{1}$ and $\epsilon_{2}$ delimits a face. Hence $e_{1}(v)$ and $e_{\mathrm{r}}(v)$ are incident to the same inner face of $D$, which implies that $v$ has degree 2 , a contradiction.

Tri-orientations. Let $D$ be a bicolored irreducible dissection and let $D^{c}$ be its completed bicolored dissection. We define a mapping $\Phi$ from the tri-orientations of $D^{c}$ to the tri-orientations of $D$. Given a tri-orientation $Y$ of $D^{c}$, we remove the edges that have been added to obtain $D^{c}$ from $D$, erase the orientation of the edges of the hexagon of $D$, and orient inward all inner half-edges incident to an outer vertex of $D$. We obtain thus a tri-orientation $\Phi(Y)$ of $D$, see the transition between Figure 13(b) and Figure 13(a).

Lemma 8.4. Let $Y$ be a tri-orientation of $D^{c}$ without clockwise circuit. Then the tri-orientation $\Phi(Y)$ of $D$ has no clockwise circuit.

For each tri-orientation $X$ of $D$ without clockwise circuit, there exists a triorientation $Y$ of $D^{c}$ without clockwise circuit such that $\Phi(Y)=X$. 


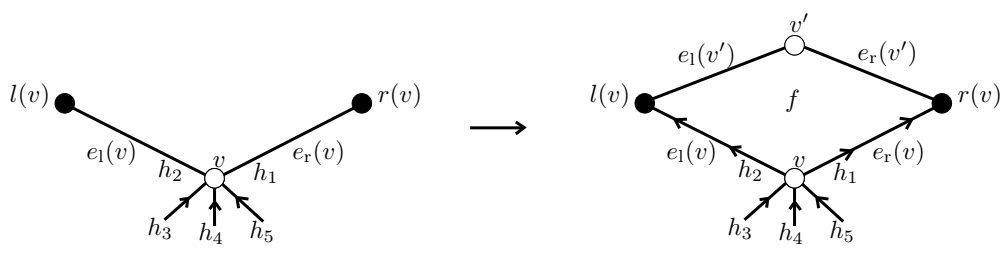

Fig. 12. From a tri-orientation $X$ of $D$ without clockwise circuit, construction of a tri-orientation $Y$ of $D^{c}$ without clockwise circuit such that $\Phi(Y)=X$.

Proof. The first point is trivial, as the tri-orientation $\Phi(Y)$ is just obtained by removing some edges and some orientations of half-edges.

For the second point, the preimage $Y$ is constructed as follows. Consider each white vertex $v$ of the hexagon of $D$ which has degree at least 3 . Let $\left(h_{1}, \ldots, h_{m}\right)$ $(m \geq 3)$ be the series of half-edges incident to $v$ in $D$ in counter-clockwise order around $v$, with $h_{1}$ and $h_{2}$ belonging respectively to the edges $e_{\mathrm{r}}(v)$ and $e_{1}(v)$. As $m \geq 3$, the vertex $v$ gives rise to a covering vertex $v^{\prime}$ with two incident edges $e_{1}\left(v^{\prime}\right)$ and $e_{\mathrm{r}}\left(v^{\prime}\right)$ such that the edges $e_{\mathrm{l}}(v), e_{\mathrm{r}}(v), e_{\mathrm{l}}\left(v^{\prime}\right)$ and $e_{\mathrm{r}}\left(v^{\prime}\right)$ form a new face $f$. The edges $e_{\mathrm{l}}(v)$ and $e_{\mathrm{r}}(v)$ become inner edges of $D^{c}$ when $v^{\prime}$ is added, and have thus to be directed.

We orient the two half-edges of $e_{1}(v)$ and $e_{\mathrm{r}}(v)$ respectively toward $l(v)$ and toward $r(v)$, see Figure 12. The vertex $v$ receives thus two outgoing half-edges, and we have to give to $v$ a third outgoing half-edge. The suitable choice to avoid the appearance of a clockwise circuit is to orient $h_{3}$ outward, see Figure 12. Indeed, assume a contrario that a simple clockwise circuit $\mathcal{C}$ is created. Then the circuit must pass by $v$. It goes into $v$ using one of the half-edges $h_{i}$ directed toward $v$, i.e., $i \geq 4$. Moreover, it must go out of $v$ using the half-edge $h_{3}$ (indeed, if the circuit uses $h_{1}$ or $h_{2}$ to go out of $v$, then it reaches an outer vertex, which has outdegree 0 ). Hence, the interior of the clockwise circuit $\mathcal{C}$ must contain all faces incident to $v$ that are on the right of $v$ when we traverse $v$ from $h_{i}$ and go out using $h_{3}$. Hence, the interior of $\mathcal{C}$ must contain the new face $f$ of $D^{c}$, see Figure 12. But $f$ is incident to outer edges of $D^{c}$, hence the clockwise circuit $\mathcal{C}$ must pass by outer edges of $D^{c}$, which are not oriented, a contradiction. Thus, we have constructed a tri-orientation $Y$ of $D^{c}$ without clockwise circuit and such that $\Phi(Y)=X$. An example of this construction can be seen as the transition between Figure 13(a) and Figure 13(b).

LEMMA 8.5. The existence and uniqueness of a tri-orientation without clockwise circuit for any bicolored complete irreducible dissection implies the existence and uniqueness of a tri-orientation without clockwise circuit for any irreducible dissection, i.e., implies Theorem 4.4.

Proof. This is a clear consequence of Lemma 8.3 and Lemma 8.4.

Complete-tri-orientations. A complete-tri-orientation of a bicolored complete irreducible dissection $D$ is an orientation of the half-edges of $D$ that satisfies the following conditions (very similar to the conditions of a tri-orientation): all black vertices and all inner white vertices of $D$ have outdegree 3 , the three white vertices 


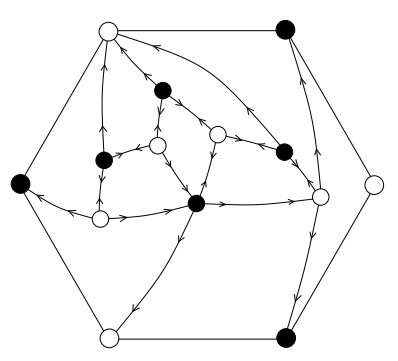

(a)

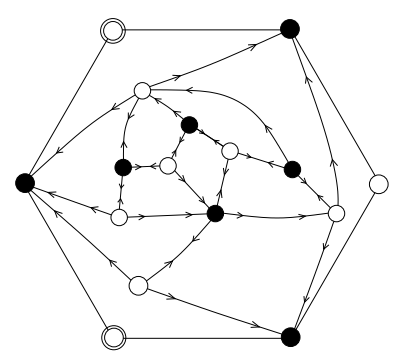

(b)

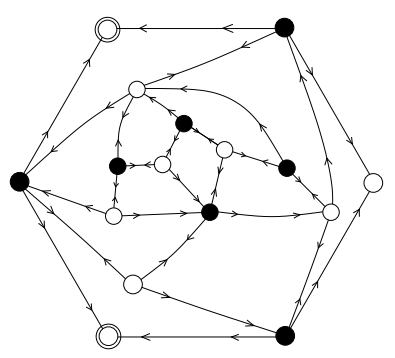

(c)

Fig. 13. A bicolored irreducible dissection $D$ endowed with a tri-orientation $X$ without clockwise circuit (Figure a). The associated completed dissection $D^{c}$ (the two added white vertices are surrounded) endowed with the tri-orientation $Y$ such that $\Phi(Y)=X$ (Figure b). The dissection $D^{c}$ endowed with the complete-tri-orientation $Z$ such that $\Psi(Z)=Y$ (Figure c).

of the hexagon have outdegree 0 , and the two half-edges of an edge of $D$ can not both be oriented inward. The difference with the definition of tri-orientation is that the half-edges of the hexagon are oriented, with prescribed outdegree for the outer vertices. Similarly as in a tri-orientation, edges of $D$ are distinguished into simply-oriented edges and bi-oriented edges.

Lemma 8.6. Let $D \in \mathcal{D}_{n}$ be a bicolored complete irreducible dissection endowed with a complete-tri-orientation without clockwise circuit. Then the subgraph $T$ of $D$ consisting of the bi-oriented edges of $D$ is a tree incident to all vertices of $D$ except the three outer white vertices.

Proof. We reason similarly as in Lemma 4.2. Let $r$ and $s$ be the numbers of bioriented and simply oriented edges of $D$. From Euler's relation (using the degrees of the faces of $D$ ), $D$ has $2 n+7$ edges, i.e., $r+s=2 n+7$. In addition, the $n$ inner vertices and the three black (resp. white) vertices of the hexagon of $D$ have outdegree 3 (resp. 0). Hence, $2 r+s=3(n+3)$. Thus, $r=n+2$ and $s=n+5$. Hence, the subgraph $T$ has $n+2$ edges, has no cycle (otherwise, a clockwise circuit of $D$ would exist), and is incident to at most $(n+3)$ vertices, which are the inner vertices and the three outer black vertices of $D$. A classical result of graph theory ensures that $T$ is a tree spanning these $(n+3)$ vertices.

Lemma 8.7. Let $D \in \mathcal{D}_{n}$ be a bicolored complete irreducible dissection endowed with a complete-tri-orientation $Z$ without clockwise circuit. Then, for each outer black vertex $v$ of $D$, the unique outgoing inner half-edge incident to $v$ belongs to a bi-oriented edge.

Proof. The subgraph $T$ consisting of the bi-oriented edges of $D$ is a tree spanning all vertices of $D$ except the three outer white vertices. Hence, there is a bi-oriented edge $e$ incident to each black vertex $v$ of the hexagon and this edge consitutes the third outgoing edge of $v$.

Let $D$ be a bicolored complete irreducible dissection and $Z$ be a complete-triorientation of $D$ without clockwise circuit. We associate to $Z$ a tri-orientation $\Psi(Z)$ as follows: erase the orientation of the edges of the hexagon of $D$; for each black 


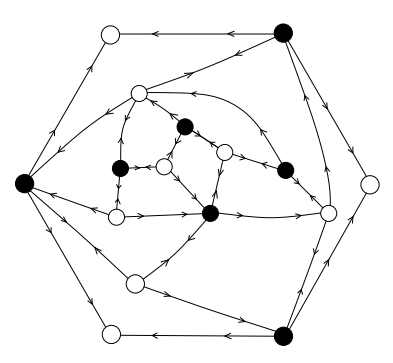

(a)

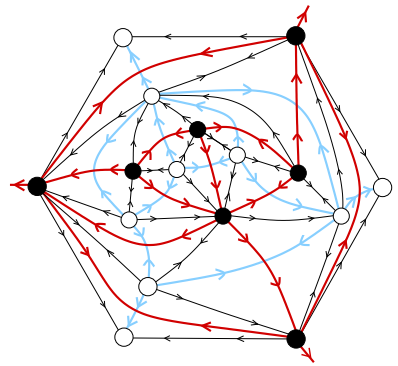

(b)

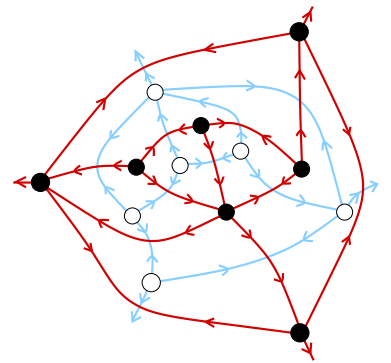

(c)

Fig. 14. The construction of the derived map of a bicolored complete irreducible dissection. The dissection is endowed with a complete-tri-orientation without clockwise circuit, and the derived map is endowed with the orientation obtained using the transposition rules for orientations.

vertex $v$ of the hexagon, change the orientation of the unique outgoing inner halfedge $h$ of $v$. According to Lemma 8.7, $h$ belongs to a bi-oriented edge $e$, so that the change of orientation of $h$ turns $e$ into an edge simply oriented toward $v$. Thus, the obtained orientation $\Psi(Z)$ is a tri-orientation.

Lемма 8.8. Let $D$ be a bicolored complete irreducible dissection. Let $Z$ be a complete-tri-orientation of $D$ without clockwise circuit. Then the tri-orientation $\Psi(Z)$ of $D$ has no clockwise circuit.

For each tri-orientation $Y$ of $D$ without clockwise circuit, there exists a completetri-orientation $Z$ of $D$ without clockwise circuit such that $\Psi(Z)=Y$.

ProOf. The first point is trivial. For the second point, we reason similarly as in Lemma 8.4. For each black vertex $v$ of the hexagon of $D$, let $\left(h_{1}, \ldots, h_{m}\right)(m \geq 3)$ be the sequence of half-edges of $D$ incident to $v$ in counter-clockwise order around $v$, with $h_{1}$ and $h_{2}$ belonging to the two outer edges $e_{\mathrm{r}}(v)$ and $e_{1}(v)$ of $D$ that are incident to $v$. To construct the preimage $Z$ of $Y$, we make the edges $e_{1}(v)$ and $e_{\mathrm{r}}(v)$ simply oriented toward their incident white vertex. The third outgoing half-edge is chosen to be $h_{3}$, which is the "leftmost" inner half-edge of $v$. An argument similar as in the proof of the second point of Lemma 8.4 ensures that this choice is judicious to avoid the creation of a clockwise circuit. An example of this construction is shown in Figure 13(b)-(c).

Finally, Proposition 8.2 follows directly from Lemma 8.5 and Lemma 8.8.

Proposition 8.5 reduces the proof of Theorem 4.4 to proving the existence and uniqueness of a complete-tri-orientation without $\mathrm{cw}$ circuit for any bicolored complete irreducible dissection. From now on, we will work with these dissections.

\subsection{Transposition rules for orientations}

Let $D$ be a bicolored complete irreducible dissection and let $G^{\prime}$ be the derived map of $D$. We associate to a complete-tri-orientation of $D$ an orientation of the edges of $G^{\prime}$ of $D$ as follows, see Figure 14: each edge $e=\left(v, v^{\prime}\right)$-with $v$ the primal/dual vertex and $v^{\prime}$ the edge-vertex - receives the direction of the half-edge of $D$ following $e$ in ccw order around $v$. 
Lemma 8.9. Let $D$ be a bicolored complete irreducible dissection endowed with a complete-tri-orientation without clockwise circuit. Then the orientation of the derived map $G^{\prime}$ of $D$ obtained using the transposition rules has the following properties:

- each primal or dual vertex of $G^{\prime}$ has outdegree 3.

- each edge-vertex of $G^{\prime}$ has outdegree 1.

In other words, the orientation of $G^{\prime}$ obtained by applying the transposition rules is an $\alpha_{0}$-orientation.

Proof. The first point is trivial. For the second point, let $f$ be an inner face of $D$ and $v_{f}$ the associated edge-vertex of $G^{\prime}$ (we recall that $v_{f}$ is the intersection of the two diagonals of $f$ ). The transposition rules for orientation ensures that the outdegree of $v_{f}$ in $G^{\prime}$ is the number $n_{f}$ of inward half-edges of $D$ incident to $f$. Hence, to prove that each edge-vertex of $G^{\prime}$ has outdegree 1, we have to prove that $n_{f}=1$ for each inner face $f$ of $D$. Observe that $n_{f}$ is a positive number, otherwise the contour of $f$ would be a clockwise circuit. Let $n$ be the number of inner vertices of $D$. Euler's relation implies that $D$ has $(n+2)$ inner faces and $(4 n+14)$ half-edges. By definition of a complete-tri-orientation, $3(n+3)$ half-edges are outgoing. Hence, $(n+5)$ half-edges are ingoing. Among these $(n+5)$ ingoing half-edges, exactly three are incident to the outer face (see Figure 13(c)). Hence, $D$ has $(n+2)$ half-edges incident to an inner face, so that $\sum_{f} n_{f}=n+2$. As $\sum_{f} n_{f}$ is a sum of $(n+2)$ positive numbers adding to $(n+2)$, the pigeonhole's principle ensures that $n_{f}=1$ for each inner face $f$ of $D$.

\subsection{Uniqueness of a tri-orientation without clockwise circuit}

The following lemma is the companion of Lemma 8.9 and is crucial to establish the uniqueness of a tri-orientation without clockwise circuit for any irreducible dissection.

LemMa 8.10. Let $D$ be a bicolored complete irreducible dissection endowed with a complete-tri-orientation $Z$ without clockwise circuit. Let $G^{\prime}$ be the derived map of $D$. Then the $\alpha_{0}$-orientation $X$ of $G^{\prime}$ obtained from $Z$ by the transposition rules has no clockwise circuit.

Proof. Assume that $X$ has a clockwise circuit $\mathcal{C}$. Each edge of $G^{\prime}$ connects an edge-vertex and a vertex of the original dissection $D$. Hence, the circuit $\mathcal{C}$ consists of a sequence of pairs $(\underline{e}, \bar{e})$ of consecutive edges of $G^{\prime}$ such that $\underline{e}$ goes from a vertex $\underline{v}$ of the dissection toward an edge-vertex $v^{\prime}$ of $G^{\prime}$ and $\bar{e}$ goes from $v^{\prime}$ toward a vertex $\bar{v}$ of the dissection. Let $\left(e_{1}^{\prime}, \ldots, e_{m}^{\prime}\right)$ be the sequence of edges of $G^{\prime}$ between $\underline{e}$ and $\bar{e}$ in clockwise order around $v^{\prime}$, so that $e_{1}^{\prime}=\underline{e}$; and $e_{m}^{\prime}=\bar{e}$ and let $\left(v_{1}, \ldots, v_{m}\right)$ be their respective extremities, so that $v_{1}=\underline{v}$ and $v_{m}=\bar{v}$. Notice that $2 \leq m \leq 4$.

As each edge-vertex has outdegree 1 in $X$ and as $e_{m}^{\prime}$ is going out of $v^{\prime}$, the edges $e_{1}^{\prime}, \ldots, e_{m-1}^{\prime}$ are directed toward $v^{\prime}$. Hence, the transposition rules for orientations ensure that the edges $\left(v_{i}, v_{i+1}\right)$, for $1 \leq i \leq m-1$, are all bi-oriented or oriented from $v_{i}$ to $v_{i+1}$ in the complete-tri-orientation $Z$ of $D$. Hence, we can go from $\underline{v}$ to $\bar{v}$ passing by the exterior of $\mathcal{C}$ and using only edges of $D$, see Figure 15 for an example, where $m=3$. 


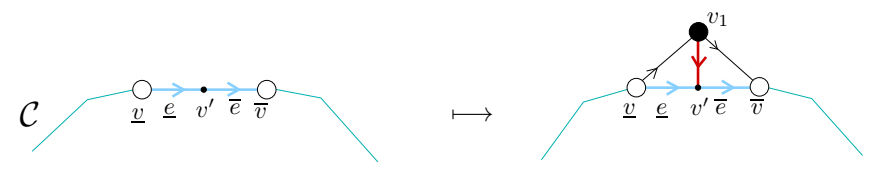

Fig. 15. An oriented path of edges of the dissection can be associated to each pair $(\underline{e}, \bar{e})$ of consecutive edges of $\mathcal{C}$ sharing an edge-vertex.

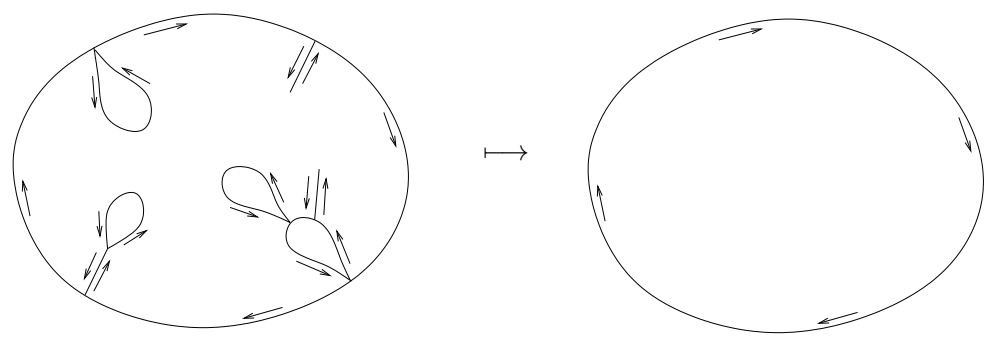

Fig. 16. A simple clockwise circuit can be extracted from an oriented path enclosing a bounded simply connected region on its right.

Concatenating the paths of edges of $D$ associated to each pair $(\underline{e}, \bar{e})$ of $\mathcal{C}$, we obtain a closed oriented path of edges of $D$ enclosing the interior of $\mathcal{C}$ on its right. Clearly, a simple clockwise circuit can be extracted from this closed path, see Figure 16. As the complete-tri-orientation $Z$ has no clockwise circuit, this yields a contradiction.

Proposition 8.11. Each irreducible dissection has at most one tri-orientation without clockwise circuit.

Proof. Let $D$ be a bicolored complete irreducible dissection and $G^{\prime}$ its derived map. A first important remark is that the transposition rules for orientations clearly define an injective mapping. In addition, Lemma 8.10 ensures that the image of a complete-tri-orientation of $D$ without clockwise circuit is an $\alpha_{0}$-orientation of $G^{\prime}$ without clockwise circuit. Hence, injectivity of the mapping and uniqueness of an $\alpha_{0}$-orientation without clockwise circuit of $G^{\prime}$ (Theorem 8.1) ensure that $D$ has at most one complete-tri-orientation without clockwise circuit. Hence, Proposition 8.2 implies that each irreducible dissection has at most one tri-orientation without clockwise circuit.

\subsection{Existence of a tri-orientation without clockwise circuit}

Inverse of the transposition rules. Let $D$ be a bicolored complete irreducible dissection and $G^{\prime}$ its derived map. Given an $\alpha_{0}$-orientation of $G^{\prime}$, we associate to this orientation an orientation of the half-edges of $D$ by performing the inverse of the transposition rules: each half-edge $h$ of $D$ receives the orientation of the edge of $G^{\prime}$ that follows $h$ in clockwise order around its incident vertex, see Figure 14(b).

Lemma 8.12. Let $D$ be an irreducible dissection and $G^{\prime}$ the derived map of $D$, endowed with its minimal $\alpha_{0}$-orientation. Then the inverse of the transposition rules for orientations yields a complete-tri-orientation of $D$.

ACM Journal Name, Vol. V, No. N, Month 20YY. 


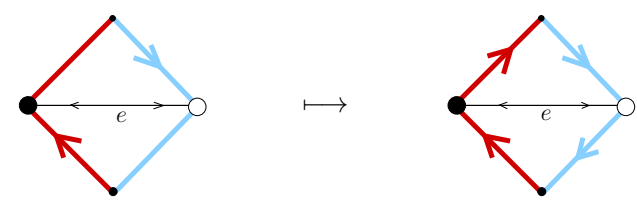

Fig. 17. The case where the two half-edges of $e$ are oriented inward implies that the boundary of the associated face of $G^{\prime}$ is a clockwise circuit.

Proof. The inverse of the transposition rules is clearly such that a vertex has the same outdegree in the orientation of $D$ as in the $\alpha_{0}$-orientation of $G^{\prime}$. Hence, each vertex of $D$ has outdegree 3 except the 3 outer white vertices that have outdegree 0 , see Figure 14(b).

To prove that the orientation of $D$ is a complete-tri-orientation, it remains to show that the two half-edges of an edge $e$ of $D$ can not both be oriented inward. Assume a contrario that there exists such an edge $e$. The transposition rules for orientation and the fact that each edge-vertex of $G^{\prime}$ has outdegree 1 imply that the boundary of the face $f_{e}$ of $G^{\prime}$ associated to $e$ is a clockwise circuit, see Figure 17 . This yields a contradiction with the minimality of the $\alpha_{0}$-orientation.

Lemma 8.13. Let $D$ be a bicolored complete irreducible dissection and let $G^{\prime}$ be its derived map. Then the complete-tri-orientation of $D$ associated with the minimal $\alpha_{0}$-orientation of $G^{\prime}$ has no cw circuit.

Proof. Let $X$ be the minimal $\alpha_{0}$-orientation of $G^{\prime}$ and let $Z$ be the associated complete-tri-orientation of $D$. Assume that $Z$ has a clockwise circuit $\mathcal{C}$. For each vertex $v$ on $\mathcal{C}$, we denote by $h_{v}$ the half-edge of $\mathcal{C}$ starting from $v$ with the interior of $\mathcal{C}$ on its right, and we denote by $e_{v}$ the edge of $G^{\prime}$ that follows $h_{v}$ in clockwise order around $v$. As $\mathcal{C}$ is a clockwise circuit for $Z, h_{v}$ is going out of $v$. Hence, by definition of the transposition rules, $e_{v}$ is going out of $v$. Observe that, in the interior of $\mathcal{C}, e_{v}$ is the most counter-clockwise edge of $G^{\prime}$ incident to $v$.

We use this observation to build iteratively a clockwise circuit of $X$, yielding a contradiction. First we state the following result proved in [Felsner 2004]: "for each vertex $v \in G^{\prime}$ there exists a simple oriented path $\mathcal{P}_{v}$ in $G^{\prime}$, called the straight path of $v$, which starts at $v$ and ends at a vertex incident to the outer face of $G^{\prime \prime}$. Let $v_{0}$ be a vertex on $\mathcal{C}$, and $\mathcal{P}_{v_{0}}$ be the straight path starting at $e_{v_{0}}$ for the orientation $X$. Then $\mathcal{P}_{v_{0}}$ has to reach $\mathcal{C}$ at a vertex $v_{1}$ different from $v_{0}$. Denote by $P_{1}$ the part of $\mathcal{P}_{v_{0}}$ between $v_{0}$ and $v_{1}$, by $\Lambda_{1}$ the part of the clockwise circuit $\mathcal{C}$ between $v_{1}$ and $v_{0}$, and by $\mathcal{C}_{1}$ the cycle enclosed by the concatenation of $P_{1}$ and $\Lambda_{1}$. Let $\mathcal{P}_{v_{1}}$ be the straight path starting at $e_{v_{1}}$. The fact that $e_{v_{1}}$ is the most counterclockwise incident edge of $v_{1}$ in the interior of $\mathcal{C}$ ensures that $\mathcal{P}_{v_{1}}$ starts in the interior of $\mathcal{C}_{1}$. Then, the path $\mathcal{P}_{v_{1}}$ has to reach $\mathcal{C}_{1}$ at a vertex $v_{2} \neq v_{1}$. We denote by $P_{2}$ the part of the path $\mathcal{P}_{v_{1}}$ between $v_{1}$ and $v_{2}$. If $v_{2}$ belongs to $P_{1}$, then the concatenation of the part of $P_{1}$ between $v_{2}$ and $v_{1}$ and of the part of $P_{2}$ between $v_{1}$ and $v_{2}$ is a clockwise circuit, a contradiction. Hence, $v_{2}$ is on $\Lambda_{1}$ strictly between $v_{1}$ and $v_{0}$. We denote by $\bar{P}_{2}$ the concatenation of $P_{1}$ and $P_{2}$, and by $\Lambda_{2}$ the part of $\mathcal{C}$ going from $v_{2}$ to $v_{0}$. As $v_{2}$ is strictly between $v_{1}$ and $v_{0}, \Lambda_{2}$ is strictly included in $\Lambda_{1}$. Finally, we denote by $\mathcal{C}_{2}$ the cycle made of the concatenation of $\bar{P}_{2}$ and $\Lambda_{2}$. Hence, 


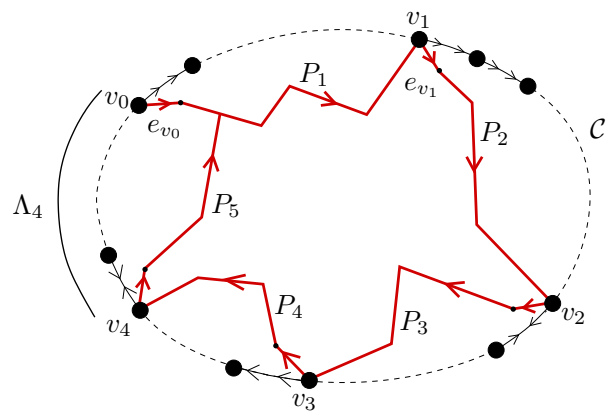

Fig. 18. The presence of a clockwise circuit in $Z$ implies the presence of a clockwise circuit in $X$.

similarly as for the path $\mathcal{P}_{v_{1}}$, the straight path $\mathcal{P}_{v_{2}}$ starting at $e_{v_{2}}$ must start in the interior of $\mathcal{C}_{2}$.

Then we continue iteratively, see Figure 18. At each step $k$, we consider the straight path $\mathcal{P}_{v_{k}}$ starting at $e_{v_{k}}$. This path starts in the interior of the cycle $\mathcal{C}_{k}$, and reaches $\mathcal{C}_{k}$ at another vertex $v_{k+1}$. This vertex $v_{k+1}$ can not belong to $\bar{P}_{k}:=P_{1} \cup \ldots \cup P_{k}$, otherwise a clockwise circuit of $X$ would be created. Hence, $v_{k+1}$ is on $\mathcal{C}$ strictly between $v_{k}$ and $v_{0}$. In particular the path $\Lambda_{k+1}$ going from $v_{k+1}$ to $v_{0}$ on $\mathcal{C}$, is strictly included in the path $\Lambda_{k}$ going from $v_{k}$ to $v_{0}$ on $\mathcal{C}$, i.e., $\Lambda_{k}$ shrinks strictly at each step. Thus, there must be a step $k_{0}$ when $\mathcal{P}_{v_{k_{0}}}$ reaches $\mathcal{C}_{k_{0}}$ at a vertex on $\bar{P}_{k_{0}}$, creating a clockwise circuit of $X$, a contradiction.

Proposition 8.14. For each irreducible dissection, there exists a tri-orientation without clockwise circuit.

ProOF. Lemma 8.13 ensures that each bicolored complete irreducible dissection $D$ has a complete-tri-orientation $Z$ without clockwise circuit; and Proposition 8.2 ensures that the existence of a complete-tri-orientation without clockwise circuit for any bicolored complete irreducible dissection implies the existence of a triorientation without clockwise circuit for any irreducible dissection.

Finally, Theorem 4.4 follows from Proposition 8.11 and Proposition 8.14.

\section{COMPUTING THE MINIMAL $\alpha_{0}$-ORIENTATION OF A DERIVED MAP}

We describe in this section a linear-time algorithm to compute the minimal $\alpha_{0^{-}}$ orientation of the derived map of an outer-triangular 3-connected plane graph. This result is crucial for the encoding algorithm of Section 7 to have linear time complexity (see the transition between Figure 11(b) and Figure 11(c)).

As discussed in [Felsner 2004], given a 3-connected map $G$ and its derived map $G^{\prime}$, an $\alpha_{0}$-orientations of $G^{\prime}$ corresponds to a so-called Schnyder wood of $G$. These Schnyder woods of 3-connected maps are the right generalisations of Schnyder woods of triangulations [Schnyder 1990]. Quite naturally, our algorithm is a generalization of the algorithm to compute the minimal Schnyder wood of a triangulation [Brehm 2000]. The ideas for the extension to 3-connected maps have already been introduced by [Kant 1996] and [di Battista et al. 1999]. The algorithm of [di Battista et al. 1999] outputs a Schnyder wood of a 3-connected map; 
which can be subsequently made minimal by iterated circuit reversions with a linear overall complexity, as easily follows from ideas presented in [Khuller et al. 1993]. Our algorithm relies on similar principles, suitably modified so as to ouput directly the minimal Schnyder wood (i.e., the Schnyder wood associated with the minimal $\alpha_{0}$-orientation), also in linear time. In itself our algorithm for 3-connected maps is only slightly more involved than the algorithm for triangulations, as opposed to the correctness proof, which is much harder (see the discussion at the beginning of Section 10). Because of this we give a rather proof-oriented description of the algorithm.

Our algorithm is also of independent interest in connection with Schnyder woods, and it has applications in the context of graph drawing. Indeed, the minimal Schnyder wood orientation is also a key ingredient for the straight-line drawing algorithm presented in [Bonichon et al. 2007]. This algorithm relies on operations of edge-deletion, embedding of the obtained graph, and then embedding of the deleted edges. The grid size is guaranteed to be bounded by $(n-2) \times(n-2)$-equalling at least Schnyder's algorithm [Schnyder 1990] — provided the Schnyder wood used is the one associated to the minimal $\alpha_{0}$-orientation. An implementation of this drawing algorithm including our orientation algorithm has been made available by Bonichon in [de Fraysseix et al. ].

\subsection{Principle of the algorithm}

Let $G$ be an outer-triangular 3-connected planar graph and let $G^{\prime}$ be its derived map and $G^{*}$ its dual map. We denote by $a_{1}, a_{2}$ and $a_{3}$ the outer vertices of $G$ in clockwise order. We describe here a linear-time iterative algorithm to compute the minimal $\alpha_{0}$-orientation of $G^{\prime}$. The idea is to maintain a simple cycle of edges of $G$; at each step $k$, the cycle, denoted by $\mathcal{C}_{k}$, is shrinked by choosing a so-called eligible vertex $v$ on $\mathcal{C}_{k}$, and by removing from the interior of $\mathcal{C}_{k}$ all faces incident to $v$. The eligible vertex is always different from $a_{2}$ and $a_{3}$, so that the edge $\left(a_{2}, a_{3}\right)$, called base-edge, is always on $\mathcal{C}_{k}$. The edges of $G^{\prime}$ ceasing to be on $\mathcal{C}_{k}$ or in the interior of $\mathcal{C}_{k}$ are oriented so that the following invariants remain satisfied.

\section{Orientation invariants:}

- For each edge $e$ of $G$ outside $\mathcal{C}_{k}$, the 4 edges of $G^{\prime}$ incident to the edge-vertex $v_{e}$ associated to $e$ have been oriented at a step $j<k$ and $v_{e}$ has outdegree 1 .

- All other edges of $G^{\prime}$ are not yet oriented.

Moreover, the edges that correspond to half-edges of $G$ also receive a label in $\{1,2,3\}$, so that the following invariants for labels remain satisfied:

\section{Labelling invariants:}

- At each step $k$, every vertex $v$ of $G$ outside of $\mathcal{C}_{k}$ has one outgoing half-edge for each label 1, 2 and 3 and these outgoing edges appear in clockwise order around $v$. In addition, all edges between the outgoing edges with labels $i$ and $i+1$ are incoming with label $i-1$, see Figure 19(a).

- Let $v$ be a vertex of $G$ on $\mathcal{C}_{k}$ having at least one incident edge of $G^{\prime}$ outside of $G_{k}$. Then exactly one of these edges, denoted by $e_{1}^{\prime}$, is going out of $v$. In addition it has label 1. The edges of $G^{\prime}$ incident to $v$ and between $e_{1}^{\prime}$ and its left neighbour 


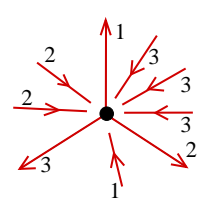

(a)

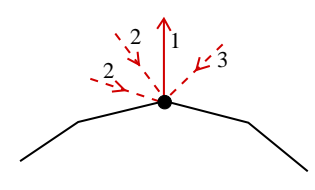

(b)

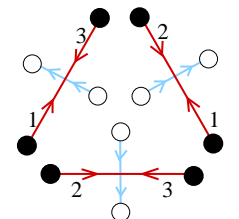

(c)

Fig. 19. The invariants for the labels of the half-edges of $G$ maintained during the algorithm.

on $\mathcal{C}_{k}$ are incoming with label 2 ; and the edges incident to $v$ in $G^{\prime}$ between $e_{1}^{\prime}$ and its right neighbour on $\mathcal{C}_{k}$ are incoming with label 3, see Figure 19(b).

- For each edge $e$ of $G$ outside of $G_{k}$, let $e^{\prime}$ be the unique outgoing edge of its associated edge-vertex $v_{e}$. Two cases can occur:

- If $e^{\prime}$ is an half-edge of $G$ then the two edges of $G^{\prime}$ incident to $v_{e}$ and forming the edge $e$ are identically labelled. This corresponds to the case where $e$ is "simply oriented".

- If $e^{\prime}$ is an half-edge of $G^{*}$, we denote by $1 \leq i \leq 3$ the label of the edge of $G^{\prime}$ following $e^{\prime}$ in clockwise order around $v_{e}$. Then the edge of $G^{\prime}$ following $e^{\prime}$ in counter-clockwise order around $v_{e}$ is labelled $i+1$, see Figure 19(c). This corresponds to the case where $e$ is "bi-oriented".

Actually, the labels are not needed to compute the orientation, but they will be very useful to prove that the algorithm outputs the minimal $\alpha_{0}$-orientation. These labels are in fact the ones of the Schnyder woods of $G$, as discussed in [Felsner 2004].

In the following, we write $G_{k}$ for the submap of $G$ obtained by removing all vertices and edges outside of $\mathcal{C}_{k}$ (at step $k$ ). In addition, we order the vertices of $\mathcal{C}_{k}$ from left to right according to the order induced by the path $\mathcal{C}_{k} \backslash\left\{a_{2}, a_{3}\right\}$, with $a_{3}$ as left extremity and $a_{2}$ as right extremity. In other words, a vertex $v \in \mathcal{C}_{k}$ is on the left of a vertex $v^{\prime} \in \mathcal{C}_{k}$ if the path of $\mathcal{C}_{k}$ going from $v$ to $v^{\prime}$ without passing by the edge $\left(a_{2}, a_{3}\right)$ has the interior of $\mathcal{C}_{k}$ on its right.

\subsection{Description of the main iteration}

Let us now describe the $k$-th step of the algorithm, during which the cycle $\mathcal{C}_{k}$ is shrinked so that the invariants for orientation and labelling remain satisfied. The description requires some definitions.

Definitions. A vertex of $\mathcal{C}_{k}$ is said to be active if it is incident to at least one edge of $G \backslash G_{k}$. Otherwise, the vertex is passive. By convention, before the first step of the algorithm, the vertex $a_{1}$ is considered as active and its incident half-edge directed toward the outer face is labelled 1.

For each pair of vertices $\left(v_{1}, v_{2}\right)$ of $\mathcal{C}_{k}$ - with $v_{1}$ is on the left of $v_{2}$-, the path on $\mathcal{C}_{k}$ going from $v_{1}$ to $v_{2}$ without passing by the edge $\left(a_{2}, a_{3}\right)$ is denoted by $\left[v_{1}, v_{2}\right]$. We also write $] v_{1}, v_{2}\left[\right.$ for $\left[v_{1}, v_{2}\right]$ deprived from the endvertices $v_{1}$ and $v_{2}$.

A pair $\left(v_{1}, v_{2}\right)$ of vertices of $\mathcal{C}_{k}$ is separating if there exists an inner face $f$ of $G_{k}$ such that $v_{1}$ and $v_{2}$ are incident to $f$ but the edges of $\left[v_{1}, v_{2}\right]$ are not all incident to $f$. Such a face is called a separating face and the triple $\left(v_{1}, v_{2}, f\right)$ is called a 
separator. The (closed) area delimited by the path $\left[v_{1}, v_{2}\right]$ and by the path of edges of $f$ going from $v_{1}$ to $v_{2}$ with the interior of $f$ on its right is called the separated area of $\left(v_{1}, v_{2}, f\right)$ and is denoted by $\operatorname{Sep}\left(v_{1}, v_{2}, f\right)$.

A vertex $v$ on $\mathcal{C}_{k}$ is said to be blocked if it belongs to a separating pair. It is easily checked that a vertex is blocked iff it is incident to a separating face of $G_{k}$. In particular, a non blocked vertex does not belong to any separating pair of vertices. By convention, the vertices $a_{2}$ and $a_{3}$ are always considered as blocked. A vertex $v$ on $\mathcal{C}_{k}$ is eligible if it is active and not blocked.

Finally, for each vertex $v$ of $\mathcal{C}_{k}$, we define its left-connection vertex $\operatorname{left}(v)$ as the leftmost vertex on $\mathcal{C}_{k}$ such that the vertices of $] \operatorname{left}(v), v$ [ all have degree 2 in $G_{k}$. The path [left $\left.(v), v\right]$ is called the left-chain of $v$ and the first edge of [left $\left.(v), v\right]$ is called the left-connection edge of $v$. Similarly, we define the right-connection vertex, the right-chain, and the right-connection edge of $v$. Notice that all vertices of $] \operatorname{left}(v), v[$ and of $] v$, right $(v)$ [ are active, as each vertex of a 3-connected graph has degree at least 3.

Operations at step $k$. First, we choose the rightmost eligible vertex of $\mathcal{C}_{k}$ and we call $v^{(k)}$ this vertex. (We will prove in Lemma 9.2 that there always exists an eligible vertex on $\mathcal{C}_{k}$ as long as $G_{k}$ is not reduced to the edge $\left(a_{2}, a_{3}\right)$.) Notice that this eligible vertex can not be $a_{2}$ nor $a_{3}$ because $a_{2}$ and $a_{3}$ are blocked.

We denote by $f_{1}, \ldots, f_{m}$ the bounded faces of $G_{k}$ incident to $v^{(k)}$ from right to left, and by $e_{1}, \ldots, e_{m+1}$ the edges of $G_{k}$ incident to $v^{(k)}$ from right to left. Hence, for each $1 \leq i \leq m, f_{i}$ corresponds to the sector between $e_{i}$ and $e_{i+1}$.

An important remark is that the right-chain of $v^{(k)}$ is reduced to one edge. Indeed, if there exists a vertex $v$ in $] v^{(k)}, \operatorname{right}\left(v^{(k)}\right)[$, then $v$ is active, as discussed above. In addition, $v$ is incident to only one inner face of $G_{k}$, namely $f_{1}$. As $f_{1}$ is incident to $v^{(k)}$ and as $v^{(k)}$ is non blocked, $f_{1}$ is not separating. Hence $v$ is not blocked. Thus $v$ is eligible and is on the right of $v^{(k)}$, in contradiction with the fact that $v^{(k)}$ is the rightmost eligible vertex on $\mathcal{C}_{k}$.

We label and orient the edges of $G^{\prime}$ incident to the edge-vertices on the left-chain of $v^{(k)}$ and on the edges $e_{1}, \ldots e_{m}$, see Figure 20:

- Inner edges: For each edge $e_{i}$ with $2 \leq i \leq m$, we denote by $v_{e_{i}}$ the corresponding edge-vertex of $G^{\prime}$. Orient the two edges of $G^{\prime}$ forming $e_{i}$ toward $v^{(k)}$ and give label 1 to these two edges. Orient the two other incident edges of $v_{e_{i}}$ toward $v_{e_{i}}$, so that $v_{e_{i}}$ has outdegree 1 .

- Left-chain: For each edge $e$ of the left-chain of $v^{(k)}$ - traversed from $v^{(k)}$ to left $\left(v^{(k)}\right)$ - different from the left-connection edge, bi-orient $e$ and give label 3 (resp. label 2) to the first (resp. second) traversed half-edge. Choose the unique outgoing edge of the edge-vertex $v_{e}$ associated to $e$ to be the edge going out of $e$ toward the interior of $\mathcal{C}_{k}$

- Left-connection edge: If left $\left(v^{(k)}\right)$ is passive, bi-orient the left-connection edge $e$ of $v^{(k)}$, give label 1 to the half-edge incident to $\operatorname{left}\left(v^{(k)}\right)$ and label 3 to the other half-edge, and choose the unique outgoing edge of the edge-vertex $v_{e}$ to be the edge going out of $v_{e}$ toward the exterior of $\mathcal{C}_{k}$. If $\operatorname{left}\left(v^{(k)}\right)$ is active, label 3 and orient toward left $\left(v^{(k)}\right)$ the two edges of $G^{\prime}$ forming $e$, and orient the two dual edges incident to $v_{e}$ toward $v_{e}$. 


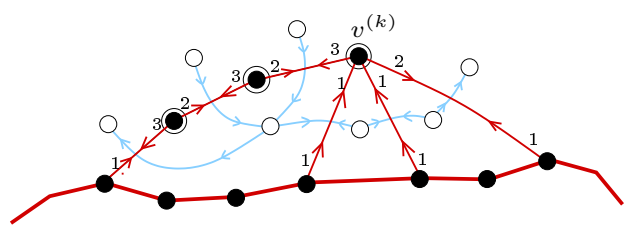

(a)

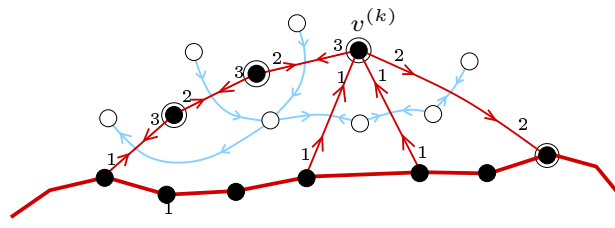

(c)

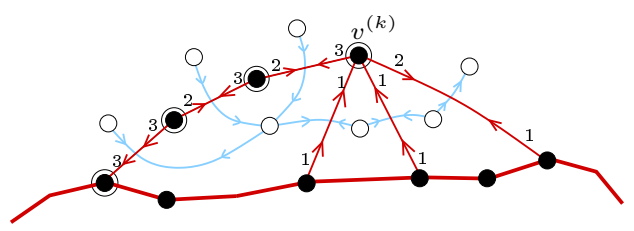

(b)

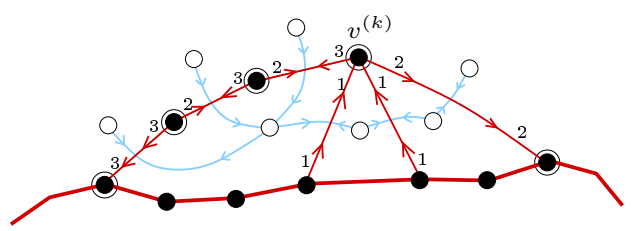

(d)

Fig. 20. The operations performed at step $k$ of the algorithm, whether $\operatorname{left}\left(v^{(k)}\right)$ and $\operatorname{right}\left(v^{(k)}\right)$ are passive-passive (Fig. a) or active-passive (Fig. b) or passive-active (Fig. c) or active-active (Fig. d). Active vertices are surrounded.

- Right-connection edge: The edge $e_{1}$, which is the right-connection edge of $v^{(k)}$, is treated symmetrically as the left-connection edge. If $\operatorname{right}\left(v^{(k)}\right)$ is passive, bi-orient $e_{1}$, give label 1 to the half-edge incident to $\operatorname{right}\left(v^{(k)}\right)$ and label 2 to the other half-edge, and choose the unique outgoing edge of the edge-vertex $v_{e_{1}}$ to be the edge going out of $v_{e_{1}}$ toward the exterior of $\mathcal{C}_{k}$. If $\operatorname{right}\left(v^{(k)}\right)$ is active, label 2 and orient toward right $\left(v^{(k)}\right)$ the two edges of $G^{\prime}$ forming $e_{1}$, and orient the two dual edges incident to $v_{e_{1}}$ toward $v_{e_{1}}$.

After these operations, all faces incident to $v^{(k)}$ are removed from the interior of $\mathcal{C}_{k}$, producing a (shrinked) cycle $\mathcal{C}_{k+1}$. As $a_{2}$ and $a_{3}$ are blocked on $\mathcal{C}_{k}, \mathcal{C}_{k+1}$ still contains the edge $\left(a_{2}, a_{3}\right)$. In addition, if $\mathcal{C}_{k+1}$ is not reduced to $\left(a_{2}, a_{3}\right)$, the property of 3-connectivity of $G$ and the fact that the chosen vertex $v^{(k)}$ is not incident to any separating face easily ensure that $\mathcal{C}_{k+1}$ is a simple cycle, i.e., it does not contain any separating vertex.

It is also easy to get convinced from Figure 19 and Figure 20 that the operations performed at step $k$ maintain the invariants of orientation and labelling.

The purpose of the next two lemmas is to prove that the algorithm terminates.

Lemma 9.1. Let $\left(v_{1}, v_{2}, f\right)$ be a separator on $\mathcal{C}_{k}$. Then there exists an eligible vertex in $] v_{1}, v_{2}[$.

Proof. Consider the (non empty) set of separators whose separated area is included or equal to the separated area of $\left(v_{1}, v_{2}, f\right)$, and let $\left(v_{1}^{\prime}, v_{2}^{\prime}, f^{\prime}\right)$ be such a separator minimal w.r.t. the inclusion of the separated areas. Observe that $v_{1}^{\prime}$ and $v_{2}^{\prime}$ are in $\left[v_{1}, v_{2}\right]$.

Assume that no vertex of $] v_{1}^{\prime}, v_{2}^{\prime}\left[\right.$ is active. Then the removal of $v_{1}^{\prime}$ and $v_{2}^{\prime}$ disconnects $\operatorname{Sep}\left(v_{1}^{\prime}, v_{2}^{\prime}, f\right)$ from $G \backslash \operatorname{Sep}\left(v_{1}^{\prime}, v_{2}^{\prime}, f\right)$. This is in contradiction with 3connectivity of $G$, because these two sets are easily proved to contain at least one vertex different from $v_{1}^{\prime}$ and $v_{2}^{\prime}$.

Hence, there exists an active vertex $v$ in $] v_{1}^{\prime}, v_{2}^{\prime}[$, also in $] v_{1}, v_{2}[$. If $v$ was incident to a separating face, this face would be included in the separated area of $\left(v_{1}^{\prime}, v_{2}^{\prime}, f^{\prime}\right)$, 
which is impossible by minimality of $\left(v_{1}^{\prime}, v_{2}^{\prime}, f^{\prime}\right)$. Hence, the active vertex $v$ is not blocked, i.e., is eligible.

Lemma 9.2. As long as $\mathcal{C}_{k}$ is not reduced to $\left(a_{2}, a_{3}\right)$, there exists an eligible vertex on $\mathcal{C}_{k}$.

Proof. Assume that there exists no separating pair of vertices on $\mathcal{C}_{k}$. In this case, an active vertex on $\mathcal{C}_{k}$ different from $a_{2}$ and $a_{3}$ is eligible. Hence we just have to prove the existence of such a vertex. At the first step of the algorithm, there exists an active vertex on $\mathcal{C}_{1} \backslash\left\{a_{2}, a_{3}\right\}$ because $a_{1}$ is active by convention. At any other step, there exists an active vertex on $\mathcal{C}_{k} \backslash\left\{a_{2}, a_{3}\right\}$, otherwise the removal of $a_{2}$ and $a_{3}$ would disconnect $G_{k} \backslash\left\{a_{2}, a_{3}\right\}$ from $G \backslash G_{k}$, in contradiction with the 3 -connectivity of $G$.

If there exists at least one separator $\left(v_{1}, v_{2}, f\right)$, Lemma 9.1 ensures that there exists an eligible vertex $v$ in $] v_{1}, v_{2}[$.

Last step of the algorithm. Lemma 9.2 implies that, at the end of the iterations, only the edge $e=\left(a_{2}, a_{3}\right)$ remains. To complete the orientation, bi-orient $e$ and label 3 (resp. label 2) the half-edge of $e$ whose origin is $a_{2}$ (resp. $a_{3}$ ); the outgoing edge of the edge-vertex $v_{e}$ (associated to $e$ ) is chosen to be the edge going out of $v_{e}$ toward the outer face. We also label respectively 2 and 3 the half-edges incident to $a_{2}$ and $a_{3}$ and directed toward the outer face.

Figure 21 illustrates the execution of the algorithm on an example, where the edges of $\mathcal{C}_{k}$ are black and bolder. In addition, the active vertices are surrounded and the rightmost eligible vertex $v^{(k)}$ is doubly surrounded.

THEOREM 9.3. The algorithm outputs the minimal $\alpha_{0}$-orientation of the derived map.

Section 10 is dedicated to the proof of this theorem.

Remark. As stated in Theorem 9.3, our orientation algorithm outputs a particular $\alpha_{0}$-orientation, namely the minimal one. The absence of clockwise circuit is due to the fact that among all eligible vertices, the rightmost one is chosen at each step. The algorithm is easily adapted to other choices of eligible vertices: the only difference is that the right-connection chain of the chosen eligible vertex might not be reduced to an edge, in which case it must be dealt with in a symmetric way as the left-connection chain (that is, 2 becomes 3 and left becomes right in the description of edge labelling and orientation). This yields a "generic" algorithm that can produce any $\alpha_{0}$-orientations of $G^{\prime}$. Indeed, given a particular $\alpha_{0}$-orientation $X$ of $G^{\prime}$, it is easy to compute a scenario (i.e., a suitable choice of the eligible vertex at each step) that outputs $X$. Such a scenario corresponds to a so-called canonical ordering for treating the vertices, see [Kant 1996].

Implementation. Following [Kant 1996] (see also [Brehm 2000] for the case of triangulations), an efficient implementation is obtained by maintaining, for each vertex $v \in \mathcal{C}_{k}$, the number $s(v)$ of separating faces incident to $v$. Thus, a vertex is blocked iff $s(v)>0$. Notice that a face $f$ is separating iff the numbers $v(f)$ and $e(f)$ of vertices and edges (except $\left.\left(a_{2}, a_{3}\right)\right)$ of $f$ belonging to $\mathcal{C}_{k}$ satisfy $v(f)>e(f)+1$. Thus, it is easy to test if a face is separating, so that the parameters $s(f)$ are also 

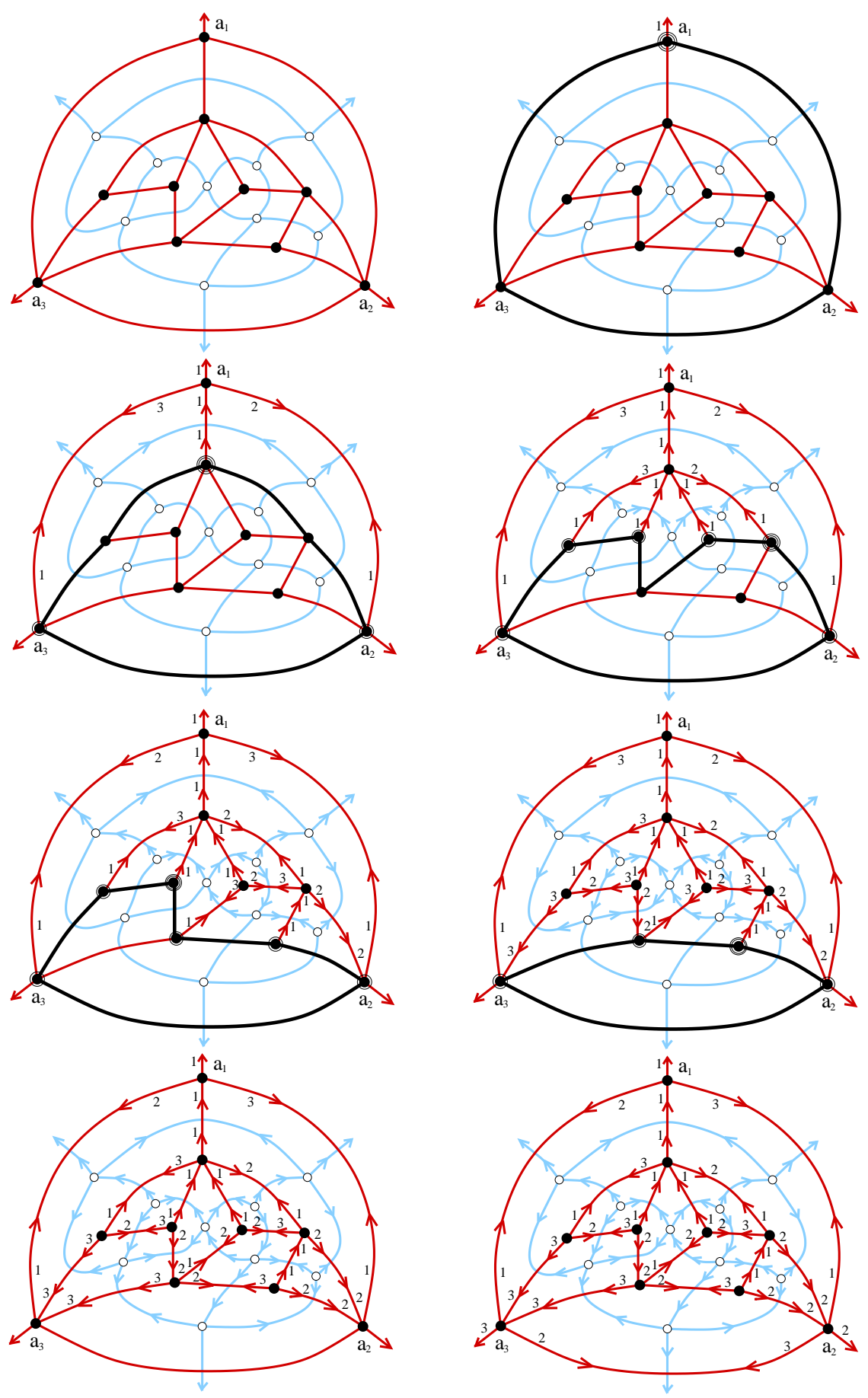

Fig. 21. The execution of the algorithm of orientation on an example. 
easily maintained. The data structure we use is the half-edge structure, which allows us to navigate efficiently on the graph. The pointer is initially on $a_{1}$, which is the rightmost eligible vertex at the first step. During the execution, once the vertex $v^{(k)}$ is treated, the pointer is moved to $v$ the right neighbour of $v^{(k)}$ on $\mathcal{C}_{k}$. The crucial point is that, if $v$ is blocked, then no vertex on the right of $v$ can be eligible (because of the nested structure of separating faces). Thus, in this case, the pointer is moved to the left until an eligible vertex is encountered. Notice also that $v$ is active after $v^{(k)}$ is treated. Thus, if $v$ is not blocked, then $v$ is eligible at step $k+1$. In this case, the nested structure of separating faces ensures that the rightmost eligible vertex at step $k+1$, if not $v$, is either the right-connection vertex $r(v)$ of $v$, or the left neighbour of $r(v)$ on $\mathcal{C}_{k+1}$ (in the case where $r(v)$ is not eligible). Notice that, in the case where $v$ is not blocked, the pointer is moved to the right but the edges traversed will be immediately treated (i.e., removed from $\left.\mathcal{C}_{k+1}\right)$ at step $k+1$. This ensures that an edge can be traversed at most twice by the pointer: once from right to left and subsequently once from left to right. Thus, the complexity is linear.

\section{PROOF OF THEOREM 9.3}

Let $G$ be an outer-triangular 3-connected map, and let $X_{0}$ be the orientation of the derived map $G^{\prime}$ computed by the orientation algorithm. This section is dedicated to proving that $X_{0}$ is the minimal $\alpha_{0}$-orientation of $G^{\prime}$.

Our proof is inspired by the proof by Brehm [2000] that ensures that, for a triangulation, the choice of the rightmost eligible vertex at each step yields the Schnyder woods without clockwise circuit. The argument is the following: the presence of a clockwise circuit implies the presence of an "inclusion-minimal" clockwise circuit which is, in the case of a triangulation, a 3 -cycle $(x, y, z)$. Then the clockwise orientation of $(x, y, z)$ determines unambiguously (up to rotation) the labels of the 3 edges of $(x, y, z)$. These labels determine an order of treatment of the 3 vertices $x$, $y$ and $z$ that is not compatible with the fact that the eligible vertex chosen at each step is the rightmost one.

In the general case of 3-connected maps, which we consider here, the proof is more involved but follows the same lines. This time there is a finite set of minimal patterns (for a triangulation this set is restricted to the triangle), such that a minimal clockwise circuit $\mathcal{C}$ in the orientation $X_{0}$ of the derived map $G^{\prime}$ can only correspond to one of these patterns (the list is shown in Figure 26). A common characteristic is that the presence of a clockwise circuit $\mathcal{C}$ for each of these patterns implies the presence of three paths $\mathcal{P}_{1}, \mathcal{P}_{2}, \mathcal{P}_{3}$ of edges of $G$ whose concatenation forms a simple cycle in $G$ (in the case of a triangulation, the three paths are reduced to one edge). In addition, the fact that $\mathcal{C}$ is clockwise determines unambiguously the labels and orientations of the edges of $\mathcal{P}_{1}, \mathcal{P}_{2}$ and $\mathcal{P}_{3}$. Writing $v_{1}, v_{2}$ and $v_{3}$ for the respective origins of these three paths, our proof (as in the case of triangulations, but with quite an amount of technical details) relies on the fact that the labels of $\mathcal{P}_{1}, \mathcal{P}_{2}, \mathcal{P}_{3}$ imply an order for processing $\left\{v_{1}, v_{2}, v_{3}\right\}$ that is not compatible with the fact that the eligible vertex chosen at each step is the rightmost one. 


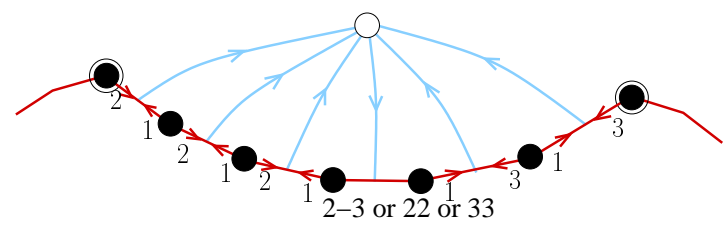

Fig. 22. The dual vertex of a face $f$ has one outgoing edge connected to the lower path of $f$.

\subsection{The algorithm outputs an $\alpha_{0}$-orientation}

By construction of the orientation, each primal vertex of the derived map $G^{\prime}$ has one outgoing edge in each label 1,2 and 3, hence it has outdegree 3. By construction also, each edge-vertex of $G^{\prime}$ has outdegree 1 . Hence, to prove that $X_{0}$ is an $\alpha_{0^{-}}$ orientation, it just remains to prove that each dual vertex of $G^{\prime}$ has outdegree 3 in $X_{0}$.

Let $f$ be an inner face of $G$ and $v_{f}$ the corresponding dual vertex in $G^{*}$. Let $k$ be the step during which $f$ is merged with the outer face of $G$. At this step, a sequence of consecutive edges of $f$ has been removed. This path of removed consecutive edges is called the upper path of $f$. The path of edges of $f$ that are not in the upper path of $f$ is called the lower path of $f$. By construction of the orientation (see Figure 20), exactly two edges of $G^{\prime}$ connecting $v_{f}$ to an edge-vertex of the upper path of $f$ are going out of $v_{f}$ : these are the edge-vertices corresponding to the two extremal edges of the upper path.

Hence it just remains to prove that exactly one edge of $G^{\prime}$ connecting $v_{f}$ to an edge-vertex of the lower path of $f$ is going out of $v_{f}$. First, observe that the lower path $P$ of $f$ is a non empty path of edges on $\mathcal{C}_{k+1}$, such that the two extremities $v_{l}$ and $v_{r}$ of the path are active and all vertices of $] v_{l}, v_{r}$ [ are passive on $\mathcal{C}_{k+1}$, see Figure 20. The fact that exactly one edge of $G^{\prime}$ connecting $v_{f}$ to an edge-vertex of $P$ is going out of $v_{f}$ is a direct consequence of the following lemma, see Figure 22.

Lemma 10.1. At a step $k$ of the algorithm, let $v_{1}$ and $v_{2}$ be two active vertices on $\mathcal{C}_{k}$ such that all vertices of $] v_{1}, v_{2}\left[\right.$ are passive. Then the path $\left[v_{1}, v_{2}\right]$ on $\mathcal{C}_{k}$ is partitioned into

- a (possibly empty) path $\left[v_{1}, v\right]$ whose edges are bi-oriented in the finally computed orientation $X_{0}$, the left half-edge having label 2 and the right half-edge label 1,

- an edge $e=\left[v, v^{\prime}\right]$ either simply oriented with label 2 from $v$ to $v^{\prime}$, or simply oriented with label 3 from $v^{\prime}$ to $v$, or bi-oriented, with label 2 on the half-edge incident to $v$ and label 3 on the half-edge incident to $v^{\prime}$,

- a (possibly empty) path $\left[v^{\prime}, v_{2}\right]$ such that, each edge of $\left[v^{\prime}, v_{2}\right]$ is bi-oriented, with label 1 on the left half-edge and label 3 on the right half-edge.

Proof. The proof is by induction on the length $L$ of $\left[v_{1}, v_{2}\right]$. Assume that $L=1$. Then $\left[v_{1}, v_{2}\right]$ is reduced to an edge. If $v_{1}$ is removed at an earlier step than $v_{2}$, then the edge $\left(v_{1}, v_{2}\right)$ is simply oriented with label 2 from $v_{1}$ to $v_{2}$. If $v_{2}$ is removed at an earlier step than $v_{1}$, then the edge $\left(v_{1}, v_{2}\right)$ is simply oriented with label 3 from $v_{2}$ to $v_{1}$. If $v_{1}$ and $v_{2}$ are removed at the same step, then $\left(v_{1}, v_{2}\right)$ is bi-oriented, with label 2 on $v_{1}$ 's side and label 3 on $v_{2}$ 's side, see Figure 20 .

ACM Journal Name, Vol. V, No. N, Month 20YY. 


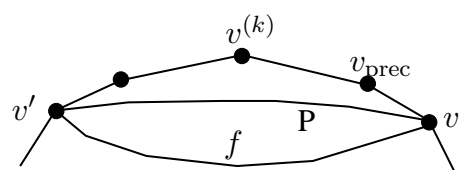

(a)

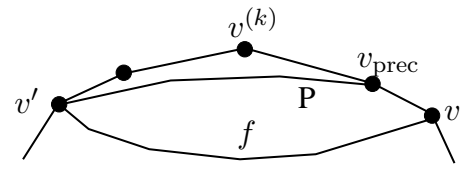

(b)

Fig. 23. The two possible configurations related to the next active vertex on the right of $v^{(k)}$.

Assume that $L>1$. Observe that the outer path $\left[v_{1}, v_{2}\right]$ remains unchanged as long as none of $v_{1}$ or $v_{2}$ is removed. This remark follows from the fact that all vertices of $] v_{1}, v_{2}$ [ are passive, so that no vertex of $\left[v_{1}, v_{2}\right]$ can be treated as long as none of $v_{1}$ or $v_{2}$ is treated.

Then, two cases can arise: if $v_{1}$ is removed before $v_{2}$, the right neighbour $v$ of $v_{1}$ becomes active and the edge $\left(v_{1}, v\right)$ is bi-oriented, with label 2 on $v_{1}$ 's side and label 1 on $v$ 's side, see Figure 20. Similarly if $v_{2}$ is removed before $v_{1}$, the left neighour $v$ of $v_{2}$ becomes active and the edge $\left(v, v_{2}\right)$ is bi-oriented with label 3 on $v_{2}$ 's side and label 1 on $v$ 's side.

The result follows by induction on $L$, with a recursive call to the path $\left[v, v_{2}\right]$ in the first case and to the path $\left[v_{1}, v\right]$ in the second case.

\subsection{The algorithm outputs the minimal $\alpha_{0}$-orientation of the derived map}

\subsubsection{Definitions and preliminary lemmas.}

Maximal bilabelled paths. Let $v$ be a vertex of $G$. For $1 \leq i \leq 3$, the $i$-path of $v$ is the unique path $P_{v}^{i}=\left(v_{0}, \ldots, v_{m}\right)$ of edges of $G$ starting at $v$ and such that each edge $\left(v_{p}, v_{p+1}\right)$ is the outgoing edge of $v_{p}$ with label $i$ (i.e., the edge of $G$ containing the outgoing half-edge of $v_{p}$ with label $i$ ). Acyclicity properties of Schnyder woods ensure that $P_{v}^{i}$ ends at the outer vertex $a_{i}$, see [Felsner 2004]. For $1 \leq i \leq 3$ and $1 \leq j \leq 3$ with $i \neq j$, we define the maximal $i-j$ path starting at $v$ as follows. Let $l \leq m$ be the maximal index such that the subpath $\left(v_{0}, \ldots, v_{l}\right)$ of $P_{v}^{i}$ only consists of bi-oriented edges with labels $i-j$. Then the maximal $i-j$ path starting at $v$ is defined to be the path $\left(v_{0}, \ldots, v_{l}\right)$ and is denoted by $P_{v}^{i-j}$.

At a step $k \geq 2$, let $v^{(k)}$ be the chosen vertex, i.e., the rightmost eligible vertex on $\mathcal{C}_{k}$. First, observe that there exists an active vertex on the right of $v^{(k)}$. Indeed, the rightmost vertex $a_{2}$ is active as soon as $k \geq 2$. In addition $a_{2}$ is non eligible on $\mathcal{C}_{k}$ because it is blocked, so that $a_{2}$ is different from $v^{(k)}$. Hence, $a_{2}$ is an active vertex on the right of $v^{(k)}$.

We define the next active vertex on the right of $v^{(k)}$ as the unique vertex $v$ on the right of $v^{(k)}$ on $\mathcal{C}_{k}$ such that all vertices of $] v^{(k)}, v[$ are passive.

Lemma 10.2. At a step $k \geq 2$, let $v^{(k)}$ be the chosen vertex. Let $v$ be the next active vertex on the right of $v^{(k)}$. Let $v_{\text {prec }}$ be the left neighbour of $v$ on $\mathcal{C}_{k}$. Then, in the orientation $X_{0}$ finally computed, each edge of $\left[v^{(k)}, v_{\text {prec }}\right]$ is bi-oriented, with label 2 on its left side and label 1 on its right side. The edge $e=\left(v_{\text {prec }}, v\right)$ is either simply oriented with label 2 from $v_{\text {prec }}$ to $v$ or bi-oriented, with label 2 on $v_{\text {prec }}$ 's side and label 3 on $v$ 's side. In other words, $P_{v^{(k)}}^{2-1}=\left[v^{(k)}, v_{\text {prec }}\right]$ and the outgoing edge of $v_{\text {prec }}$ with label 2 is $\left(v_{\text {prec }}, v\right)$. 


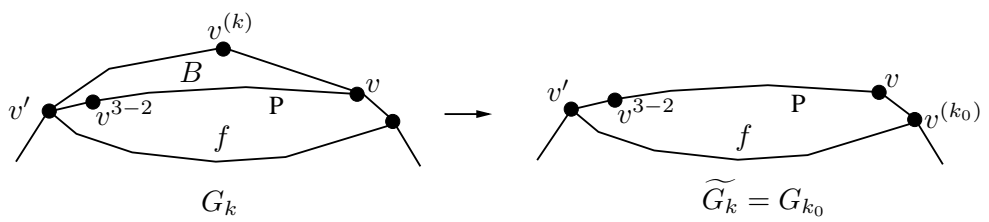

Fig. 24. The path between $v$ and $v^{3-2}$ will consist of bi-oriented edges bilabelled 3-2.

Proof. To prove this lemma, using the result of Lemma 10.1, we just have to prove that $\left(v_{\text {prec }}, v\right)$ is neither bi-oriented with label 1 on $v_{\text {prec }}$ 's side and label 3 on $v$ 's side, nor simply oriented with label 3 from $v$ to $v_{\text {prec }}$, see Figure 22.

First, as the active vertex $v$ is on the right of $v^{(k)}$, it can not be eligible, so that $v$ is blocked. As a consequence there exists a vertex $v^{\prime}$ and a face $f$ such that $\left(v, v^{\prime}, f\right)$ is a separator. Lemma 9.1 ensures that there exists an eligible vertex in ]$v^{\prime}, v\left[\right.$. Hence the vertex $v^{\prime}$ is on the left of $v^{(k)}$ on $\mathcal{C}_{k}$, otherwise $v^{(k)}$ would not be the rightmost eligible vertex. Let $P$ be the path on the boundary of $f$ going from $v$ to $v^{\prime}$ with $f$ on its left. Two cases can arise:

(1) the first edge of $P$ is different from $\left(v, v_{\text {prec }}\right)$, so that $v_{\text {prec }}$ is above $P$, see Figure 23(a). Clearly, $v$ remains blocked as long as all vertices above $P$ have not been treated. Hence, $v_{\text {prec }}$ will be treated at an earlier step that $v$. As $v$ is active, it implies (see Figure 20 ) that $\left(v_{\text {prec }}, v\right)$ is simply oriented with label 2 from $v_{\text {prec }}$ to $v$.

(2) the first edge of $P$ is $\left(v, v_{\text {prec }}\right)$, see Figure 23(b). Observe that $v_{\text {prec }}$ can not be equal to $v^{\prime}$. Indeed $v$ is on the right of $v^{(k)}$, so that $v_{\text {prec }}$ is on the right or equal to $v^{(k)}$, whereas $v^{\prime}$ is on the left of $v^{(k)}$. Hence, $P$ has length greater than 1 . As a consequence, when $f$ will cease to be separating, $v_{\text {prec }}$ will only be incident to $f$. Figure 20 ensures that, when such a vertex is treated, the edge connecting this vertex to its right neighbour is always bi-oriented and bi-labelled 2-3, which concludes the proof.

Lemma 10.3. At a step $k \geq 2$, let $v^{(k)}$ be the rightmost eligible vertex and $v$ the next active vertex on the right of $v^{(k)}$. Let $v^{3-2}$ be the extremity of $P_{v}^{3-2}$ in $X_{0}$ and $e$ the outgoing edge of $v^{3-2}$ with label 3. If $e$ is bi-oriented, it is bi-labelled 3-1 and we define $v_{1}=v^{3-2}$. Otherwise $e$ is simply oriented, we define $v_{1}$ as the extremity of $e$.

Then $v_{1}$ belongs to $\mathcal{C}_{k}$ and is on the left of $v^{(k)}$.

Proof. First, observe that each vertex $v^{\prime \prime}$ such that the pair $\left\{v^{\prime \prime}, v\right\}$ is separating is on the left of $v^{(k)}$, otherwise, Lemma 9.1 ensures that there exists an eligible vertex in $] v^{\prime \prime}, v\left[\right.$, in contradiction with the fact that $v^{(k)}$ is the rightmost eligible vertex.

Observe also that the set $\mathcal{S}$ of separators $\left(v^{\prime \prime}, v, f\right)$ involving $v$ and endowed with the inclusion-relation for the separated areas is not only a partial order but a total order. In particular, for two separators $\left(v_{1}^{\prime \prime}, v, f_{1}\right)$ and $\left(v_{2}^{\prime \prime}, v, f_{2}\right)$, if $v_{1}^{\prime \prime}$ is on the left of $v_{2}^{\prime \prime}$, then the separated area of $\left(v_{2}^{\prime \prime}, v, f_{1}\right)$ is strictly included in the separated area of $\left(v_{1}^{\prime \prime}, v, f_{2}\right)$. In addition, $\mathcal{S}$ is non empty because $v$ is the next active vertex on the right of $v^{(k)}$, hence $v$ is blocked.

ACM Journal Name, Vol. V, No. N, Month 20YY. 
Let $\left(v^{\prime}, v, f\right)$ be the maximal separator for the totally ordered set $\mathcal{S}$. Then the separated area of $\left(v^{\prime}, v, f\right)$ contains all separating faces incident to $v$ except $f$. Let $P$ be the path of edges on the boundary of $f$ going from $v$ to $v^{\prime}$ with the interior of $f$ on its left, and let $B$ be the separated area of $\left(v^{\prime}, v, f\right)$. Let $\widetilde{G_{k}}$ be the submap of $G$ obtained by removing $B$ from $G_{k}$, and let $\widetilde{\mathcal{C}_{k}}$ be the boundary of $\widetilde{G_{k}}$.

We claim that $f$ is not separating in $\widetilde{G_{k}}$. Otherwise, there would exist a vertex $v_{2}$ on the right of $v$ such that $\left(v, v_{2}, f\right)$ is a separator or there would exist a vertex $v_{3}$ on the left of $v^{\prime}$ such that $\left(v_{3}, v^{\prime}, f\right)$ is a separator: the first case is in contradiction with the fact that all separators $\left\{v, v_{2}\right\}$ involving $v$ are such that $v$ is on the right of $v_{2}$. The second case is in contradiction with the fact that $\left(v^{\prime}, v, f\right)$ is the maximal separator involving $v$.

We claim that only vertices of $B$ will be removed from step $k$ on, until all vertices of $B$ are removed. Indeed, all separating faces incident to vertices on the right of $v$ are faces of $\widetilde{G_{k}}$, hence they will remain separating as long as not all vertices of $B$ are removed. As all vertices on the right of $v$ are either blocked or passive, it is easy to see inductively that all these vertices will keep the same status until all vertices of $B$ are removed.

Let $k_{0}$ be the first step where all vertices of $B$ have been removed. Then $G_{k_{0}}=$ $\widetilde{G_{k}}$. Hence $f$ is not separating anymore on $\mathcal{C}_{k_{0}}$, but all other faces of $\widetilde{G_{k}}$ that are separating at step $k$ are still separating at step $k_{0}$. We have seen that the separating faces incident to $v$ at step $k$ are the face $f$ and faces in $B$. In addition, all faces of $G_{k_{0}}$, except $f$, have kept their separating-status between step $k$ and step $k_{0}$. Hence $v$ is eligible on $\mathcal{C}_{k_{0}}$, and the rightmost eligible vertex $v^{\left(k_{0}\right)}$ at step $k_{0}$ is a vertex incident to $f$. It is either $v$ or a vertex of $f$ on the right of $v$ (on $\mathcal{C}_{k_{0}}$ ) such that $\left[v, v^{\left(k_{0}\right)}\right]$ only consists of edges incident to $f$ (otherwise $f$ would be separating), see Figure 24, where $v^{\left(k_{0}\right)}$ is the right neighbour of $v$.

Moreover, the left-connection vertex of $v^{\left(k_{0}\right)}$ is $v^{\prime}$. Otherwise there would be a vertex of $f$ on $\widetilde{\mathcal{C}_{k}}$ and on the left of $v^{\prime}$. This vertex would also be on $\mathcal{C}_{k}$ (because only vertices of $B$ are removed to obtain $\widetilde{G_{k}}$ from $G_{k}$ ), in contradiction with the fact that $\left(v^{\prime}, v, f\right)$ is the maximal separator of $\mathcal{C}_{k}$ involving $v$.

Then two cases can arise whether $v^{\prime}$ is passive or active on $\mathcal{C}_{k_{0}}$ :

(1) $v^{\prime}$ is passive on $\mathcal{C}_{k_{0}}$. Then $v^{\prime}$ is not incident to any edge of $G \backslash G_{k_{0}}$. In particular $v^{\prime}$ is not incident to any edge of $B \backslash G_{k_{0}}$. Hence the right neighbour of $v^{\prime}$ on $\mathcal{C}_{k_{0}}$ and on $\mathcal{C}_{k}$ are the same vertex, that is, the vertex $v_{1}$ preceding $v^{\prime}$ on $P$. Observe that $v_{1}$ is on the left of $v^{(k)}$ on $\mathcal{C}_{k}$, indeed, $v_{1}$ can not be equal to $v^{(k)}$ at step $k$ because $v_{1}$ is incident to $f$, which is separating at this step. By definition of $v_{1}$ and by construction of the orientation (see Figure 20), $P_{v^{\left(k_{0}\right)}}^{3-2}$ is equal to $\left[v_{1}, v^{\left(k_{0}\right)}\right]$ taken from right to left, and $\left(v_{1}, v^{\prime}\right)$ is bi-oriented bi-labelled $3-1$ from $v_{1}$ to $v^{\prime}$. As $v \in\left[v_{1}, v^{\left(k_{0}\right)}\right]$ at step $k_{0},\left[v, v^{\left(k_{0}\right)}\right] \subseteq\left[v_{1}, v^{\left(k_{0}\right)}\right]$, so that $P_{v}^{3-2}$ is equal to $\left[v, v^{\left(k_{0}\right)}\right]$ taken from right to left. As $\left(v_{1}, v^{\prime}\right)$ is bi-oriented bi-labelled $3-1$ from $v_{1}$ to $v^{\prime}$, this concludes the proof for the first case (i.e., $v_{1}=v^{3-2}$ ).

(2) $v^{\prime}$ is active on $\mathcal{C}_{k_{0}}$. In this case, upon taking $v_{1}$ to be the vertex $v^{\prime}$, a similar argument as for the previous paragraph applies: indeed $v_{1}$ is a vertex on $\mathcal{C}_{k}$ on the left of $v^{(k)}$, and $P_{v}^{3-2}$ is the path on $\mathcal{C}_{k_{0}}$ going from $v$ to the right neighbour of $v_{1}$ on $\mathcal{C}_{k_{0}}$, and the edge connecting the right neighbour of $v_{1}$ to $v_{1}$ is simply oriented with label 3 toward $v_{1}$ (see Figure 20). 


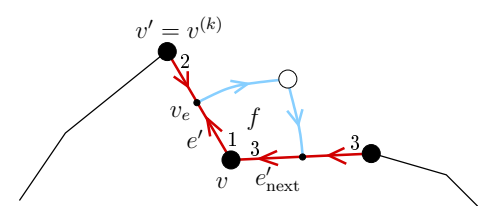

(a)

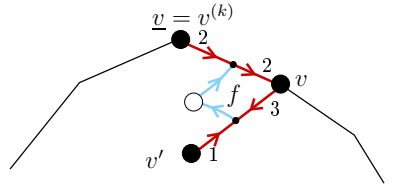

(b)

Fig. 25. Configuration of a face $f$ of $G^{\prime}$ whose boundary is a clockwise circuit and such that the outgoing edge of the unique primal vertex of $f$ has label 1 (Fig. a) and label 3 (Fig. b).

Lemma 10.4. The vertices $a_{1}, a_{2}$ and $a_{3}$ can not belong to any clockwise circuit.

Proof. Let us consider $a_{1}$ (the cases of $a_{2}$ and $a_{3}$ can be dealt with identically). The outgoing edge of $a_{1}$ with label 1 is directed toward the outer face. The outgoing edges of $a_{1}$ with labels 2 and 3 connect respectively $a_{1}$ to two edge-vertices whose unique outgoing edge is directed toward the outer face. Hence each directed path starting at $a_{1}$ finishes immediately in the outer face.

\subsubsection{Possible configurations for a minimal clockwise circuit of $X_{0}$}

LEMMA 10.5. Let $f$ be an inner face of $G^{\prime}$. Then the boundary of $f$ is not a clockwise circuit in $X_{0}$.

Proof. Assume that the contour of $f$ is a clockwise circuit. We recall that the contour of $f$ has two edge-vertices, one dual vertex, and one primal vertex $v$. Let $i$ be the label of the edge $e^{\prime}$ of $f$ going out of $v$. The edge $e^{\prime}$ is the first half-edge of an edge $e$ of $G$. We denote by $v_{e}$ the edge-vertex of $G^{\prime}$ associated to $e$ and by $v^{\prime}$ the vertex of $G$ such that $e=\left(v, v^{\prime}\right)$. As the contour of $f$ is a clockwise circuit, the unique outgoing edge of $v_{e}$ follows the edge $\left(v_{e}, v\right)$ in ccw order around $v_{e}$. Hence, according to Figure 19(c), the edge $e$ is bi-oriented and the second half-edge of $e$ has label $i+1$. We denote by $e_{\text {next }}$ the edge of $G$ following $e$ in clockwise order around $v$. The edge $e_{\text {next }}^{\prime}$ of $G^{\prime}$ following $e^{\prime}$ in clockwise order around $v$ is the edge of $f$ directed toward $v$. Hence, the rules of labelling (Figure 19(a)) ensure that $e_{\text {next }}^{\prime}$ has label $i-1$. As $e_{\text {next }}^{\prime}$ is the second half-edge of $e_{\text {next }}$, this ensures that $e_{\text {next }}$ is simply oriented with label $i-1$ toward $v$.

We now deal separately with the three possible cases $i=1,2,3$ :

- Case $i=1$ : The edge $e$ is bi-labelled 1-2 from $v$ to $v^{\prime}$ and $e_{\text {next }}$ is simply oriented with label 3 toward $v$, see Figure $25(\mathrm{a})$. Let $k$ be the step of the algorithm during which the vertex $v^{\prime}$ is treated. Figure 20 ensures that, if $v^{\prime}$ is not equal to the rightmost eligible vertex $v^{(k)}$, then the outgoing edge with label 2 of $v^{\prime}$ is bi-oriented with label 3 on the other half-edge, which is not the case here. Hence $v^{\prime}=v^{(k)}$.

In addition, as $\left(v^{\prime}, v\right)$ is bi-labelled 2-1 from $v^{\prime}$ to $v$, the vertex $v$ is passive on $\mathcal{C}_{k}$. Hence, writing $e_{v \rightarrow}$ for the edge of $\mathcal{C}_{k}$ whose left extremity is $v$, there is no edge of $G \backslash G_{k}$ between $e$ and $e_{v \rightarrow}$ in clockwise order around $v$, so that $e_{v \rightarrow}=e_{\text {next }}$.

We claim that $k \geq 2$. Otherwise $v^{\prime}$ would be equal to $a_{1}$. As $e=\left(v, v^{\prime}\right)$ is bi-labelled 1-2 from $v$ to $v^{\prime}, v$ would be equal to $a_{2}$. But according to Lemma 10.4, $a_{2}$ can not belong to any clockwise circuit.

ACM Journal Name, Vol. V, No. N, Month 20YY. 

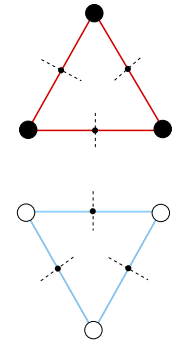

(a) $\begin{aligned} n_{e}^{(3)} & =3 \\ n_{e}^{(4)} & =0\end{aligned}$

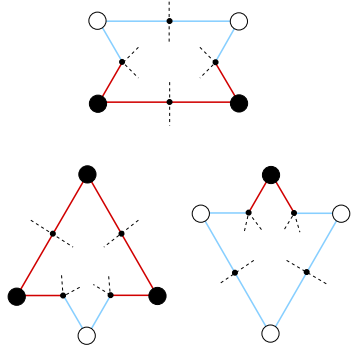

(b) $\begin{aligned} & n_{e}^{(3)}=2 \\ & n_{e}^{(4)}=2\end{aligned}$
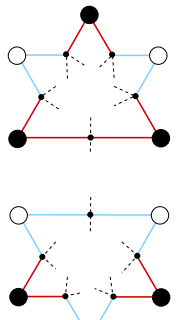

(c) $\begin{aligned} & n_{e}^{(3)}=1 \\ & n_{e}^{(4)}=4\end{aligned}$

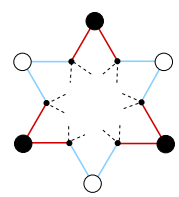

(d) $\begin{aligned} & n_{e}^{(3)}=0 \\ & n_{e}^{(4)}=6\end{aligned}$

Fig. 26. The possible configurations for a minimal clockwise circuit of $X_{0}$.

Hence $k \geq 2$ and we can use Lemma 10.2. In particular, this lemma ensures that $e_{v \rightarrow}$ is the outgoing edge of $v$ with label 2 . We obtain here a contradiction with the fact that $e_{\text {next }}$ is going toward $v$ with label 3 and $e_{v \rightarrow}=e_{\text {next }}$.

- Case $i=2$ : The edge $e$ is bi-labelled 2-3 from $v$ to $v^{\prime}$ and $e_{\text {next }}$ is simply oriented with label 1 toward $v$. Let $k$ be the step during which $v$ is treated. By construction of the orientation (see Figure 20), at step $k$ the vertex $v$ belongs to $] \operatorname{left}\left(v^{(k)}\right), v^{(k)}$ [ and $e_{\text {next }}$ is the outgoing edge of $v$ with label 3. This is in contradiction with the fact that $e_{\text {next }}$ is simply oriented toward $v$ with label 1 .

- Case $i=3$ : The edge $e$ is bi-labelled 3-1 from $v$ to $v^{\prime}$ and $e_{\text {next }}$ is simply oriented with label 2 toward $v$, see Figure 25(b). Let $\underline{v}$ be the origin of $e_{\text {next }}$ and let $k$ be the step during which $\underline{v}$ is removed from $G_{k}$. As $e_{\text {next }}$ is simply oriented with label 2 from $\underline{v}$ to $v$, we have $\underline{v}=v^{(k)}$ and $v=\operatorname{right}\left(v^{(k)}\right)$. Lemma 10.2 ensures that $v$ is the next active vertex on the right of $v^{(k)}$ on $\mathcal{C}_{k}$. In addition, $k \geq 2$, otherwise $v^{(k)}=a_{1}$, in contradiction with the fact that the outgoing edge of $a_{1}$ with label 2 is bi-oriented. Hence, we can use Lemma 10.3: here, the next active vertex on the right of $v^{(k)}$ is $v$ and the path $P_{v}^{3-2}$ is empty because the outgoing edge with label 3 of $v$ is bi-labelled 3-1. Hence the vertex denoted by $v_{1}$ in the statement of Lemma 10.3 is here $v$. Lemma 10.3 ensures that $v$ is a vertex of $\mathcal{C}_{k}$ on the left of $v^{(k)}$, in contradiction with the fact that $v$ is the right neighbour of $v^{(k)}$ on $\mathcal{C}_{k}$.

LEMma 10.6 [FELSNER 2004]. The possible configurations of an essential circuit of $X_{0}$ are illustrated in Figure 26, where $n_{e}^{(3)}$ (resp. $n_{e}^{(4)}$ ) denotes the numbers of edge-vertices on the circuit that have respectively 3 (resp. 4) incident edges on or inside the circuit.

Proof. Felsner [2004, Lem.17] shows that an essential circuit $\mathcal{C}$ of an $\alpha_{0}$-orientation has no edge in its interior whose origin is on $\mathcal{C}$. In addition, if $\mathcal{C}$ is not the boundary of a face, he shows that all edge-vertices have either one incident edge or two incident edges inside $\mathcal{C}$, which implies that the length of $\mathcal{C}$ is $6,8,10$, or 12 . The only possible configurations are those listed in Figure 26. As $X_{0}$ has no clockwise circuit of length 4 according to Lemma 10.5, this concludes the proof.

10.2.3 No configuration of Figure 26 can be a clockwise circuit in $X_{0}$. We have restricted the number of possible configurations for a clockwise circuit of $X_{0}$ to 
the list represented in Figure 26. In this section, we describe a method ensuring that the presence of a clockwise circuit for each configuration of Figure 26 yields a contradiction. The method relies on Lemma 10.2, Lemma 10.3, and on the following lemma:

Lemma 10.7. At a step $k$, let $v$ and $v^{\prime}$ be two vertices on $\mathcal{C}_{k}$ such that $v$ is on the left of $v^{\prime}$. Assume that there exists a path $P=\left(v_{0}, \ldots, v_{l}\right)$ of edges of $G$ such that $v_{0}=v, v_{l}=v^{\prime}$, and for each $0 \leq i \leq l-1$, the edge $\left(v_{i}, v_{i+1}\right)$ is the outgoing edge of $v_{i}$ with label 1 in $X_{0}$. Then $P=\left[v, v^{\prime}\right]$ on $\mathcal{C}_{k}$ and all edges of $P$ are bi-oriented bilabelled 1-3.

Proof. Proving that $P=\left[v, v^{\prime}\right]$ comes down to proving that all edges of $P$ are on $\mathcal{C}_{k}$. By construction of the orientation (see Figure 20), for each vertex $w$ of $G$, the extremity $w_{1} \in G$ of the outgoing edge of $w$ with label 1 is removed at an earlier step than $w$. Moreover, a vertex in $G \backslash G_{k}$ is removed at a step $j<k$. Hence, if $w$ is in $G \backslash G_{k}$, then $w_{1}$ is also in $G \backslash G_{k}$. Hence, if $P$ passes by a vertex outside of $G_{k}$, it can not reach $\mathcal{C}_{k}$ again. By definition of an active vertex of $\mathcal{C}_{k}$, the extremity of its outgoing edge with label 1 is a vertex of $G \backslash G_{k}$. Hence none of the vertices $v_{0}, \ldots v_{l-1}$ can be active, otherwise $P$ would pass by a vertex outside of $G_{k}$ and could not reach $\mathcal{C}_{k}$ again.

Hence, all vertices of $\mathcal{C}_{k}$ encountered by $P$ before reaching $v^{\prime}$ are passive. It just remains to prove that the outgoing edge with label 1 of each passive vertex of $\mathcal{C}_{k}$ is an edge of $\mathcal{C}_{k}$ and will be bi-oriented and bilabelled 1-3 in $X_{0}$.

Let $w$ be a passive vertex of $\mathcal{C}_{k}$ and let $w_{l}$ and $w_{r}$ be respectively the left and the right neighbour of $w$ on $\mathcal{C}_{k}$. We claim that the outgoing edge of $w$ with label 1 is the edge $\left(w, w_{l}\right)$ if $w_{l}$ will be removed before $w_{r}$ and is the edge $\left(w, w_{r}\right)$ if $w_{r}$ will be removed before $w_{l}$. Indeed, as long as none of $w_{l}$ or $w_{r}$ is removed, $w$ remains passive and keeps $w_{l}$ and $w_{r}$ as left and right neighbour. Let $k_{0}$ be the first step where $w_{l}$ or $w_{r}$ is removed. By construction of the orientation, two vertices $v_{1}$ and $v_{2}$ on the boundary of $\mathcal{C}_{k_{0}}$ such that $] v_{1}, v_{2}$ [ contains a passive vertex can not be removed at the same step. Hence, at step $k_{0}$, either $w_{l}$ or $w_{r}$ is removed. Assume that the removed vertex at step $k_{0}$ is $w_{l}$. Then, at step $k_{0},\left(w, w_{l}\right)$ is given a biorientation and receives label 1 on $w$ 's side and label 2 on $w_{l}$ 's side, see Figure 20. Similarly, if the removed vertex is $w_{r}$ then, at step $k_{0},\left(w, w_{r}\right)$ is bi-orientated and receives label 1 on $w$ 's side and label 3 on $w_{r}$ 's side.

Finally, it is easy to see that only this second case can happen in the path $P$, because the starting vertex of $P$ is on the left of the end vertex of $P$ on $\mathcal{C}_{k}$.

Lemma 10.8. None of the configurations of Figure 26 can be the boundary of a clockwise circuit in $X_{0}$.

Proof. We take here the example of the third configuration of the case $\left\{n_{e}^{(3)}=\right.$ $\left.2, n_{e}^{(4)}=2\right\}$ of Figure 26 and show why this configuration can not be a clockwise circuit in $X_{0}$. Let $\mathcal{C}$ be a clockwise circuit corresponding to such a configuration. Then $\mathcal{C}$ contains two successive dual edges $e_{1}^{*}$ and $e_{2}^{*}$-in counter-clockwise order around $\mathcal{C}$ - and a unique primal vertex which we denote by $v_{\mathcal{C}}$. Let $M^{\prime}$ be the submap of $G^{\prime}$ obtained by removing all edges and vertices outside of $\mathcal{C}$. Let $M$ be the submap of $G$ obtained by keeping only the edges whose associated edge-vertex belongs to $M^{\prime}$ and by keeping the vertices incident to these edges. As $\mathcal{C}$ is an 


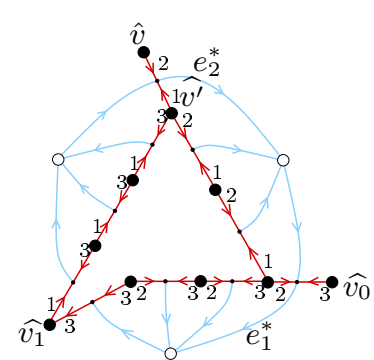

(a)

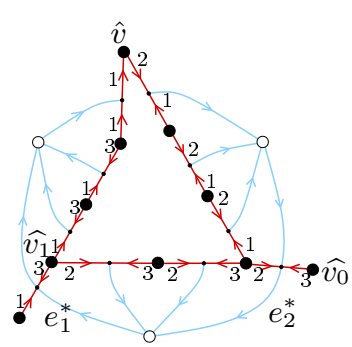

(b)

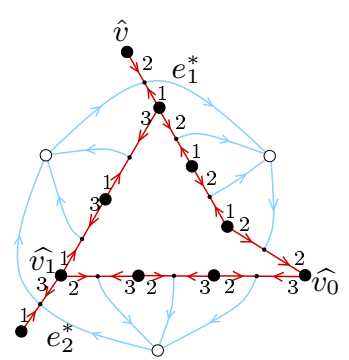

(c)

Fig. 27. The 3 possible cases for the boundary of the map $M$ associated to the third configuration of the case $\left\{n_{e}^{(3)}=2, n_{e}^{(4)}=2\right\}$ in Figure 26 .

essential circuit, no edge inside $\mathcal{C}$ has its origin on $\mathcal{C}$, see [Felsner 2004, Lem.17]. The rules of labelling (see Figure 19), the fact that all edge-vertices have outdegree 1, and the fact that no edge goes from a vertex of $\mathcal{C}$ toward the interior of $\mathcal{C}$ determine unambiguously the labels and orientations of all the edges on the boundary of $M$ in $X_{0}$, up to the label of the outgoing edge of $v_{\mathcal{C}}$ on $\mathcal{C}$. Figures $27(\mathrm{a}), 27(\mathrm{~b})$ and $27(\mathrm{c})$ represent the respective configurations when the label of the outgoing edge of $v_{\mathcal{C}}$ on $\mathcal{C}$ is 1,2 or 3 .

First, we deal with the case of Figure $27(\mathrm{a})$. Let $\hat{v}$ (resp. $\widehat{v_{0}}$ ) be the primal vertex outside of $\mathcal{C}$ and adjacent to the edge-vertex associated to $e_{2}^{*}$ (resp. $e_{1}^{*}$ ). Let $\widehat{v^{\prime}}$ be the primal vertex inside of $\mathcal{C}$ and adjacent to the edge-vertex associated to $e_{2}^{*}$. Let $k$ be the step at which $\hat{v}$ is treated. As already explained in preceding proofs (for example in the proof of Lemma 10.5), it is easy to see that $k \geq 2$ and that $\hat{v}$ is the chosen vertex $v^{(k)}$. Hence we can use Lemma 10.2 and Lemma 10.3. Lemma 10.2 and the configuration of Figure 27(a) ensure that $\widehat{v^{\prime}}$ is the right neighbour of $\hat{v}$ on $\mathcal{C}_{k}$ and that $\widehat{v_{0}}$ is the next active vertex on the right of $\hat{v}$ on $\mathcal{C}_{k}$. Moreover, the configuration of Figure 27(a) ensures that $\widehat{v_{1}}$ corresponds to the vertex $v_{1}$ in the statement of Lemma 10.3. Hence Lemma 10.3 ensures that $\widehat{v_{1}}$ is on $\mathcal{C}_{k}$ on the left of $\hat{v}$. We see on Figure 27(a) that there is an oriented path $P$ going from $\widehat{v_{1}}$ to $\widehat{v}$ such that each edge of the path is leaving with label 1 . Lemma 10.7 ensures that all edges of $P$ are bilabelled 1-3, in contradiction with the fact that $\left(\widehat{v}^{\prime}, v\right)$ is bilabelled 1-2.

We deal with the case of Figure 27(b) similarly. We define $\hat{v}:=v_{\mathcal{C}}$ and denote by $\widehat{v_{0}}$ the primal vertex outside of $\mathcal{C}$ and adjacent to the edge-vertex associated to $e_{2}^{*}$. We denote by $\widehat{v_{1}}$ the primal vertex inside of $\mathcal{C}$ and adjacent to the edge-vertex associated to $e_{1}^{*}$. Let $k$ be the step where $\hat{v}$ is removed. Then it is easy to see that $k \geq 2$ and $\hat{v}=v^{(k)}$. Hence we can use Lemma 10.2 and Lemma 10.3. Lemma 10.2 and the configuration of Figure 27(b) ensure that $\widehat{v_{0}}$ is the next active vertex on the right of $\hat{v}$ on $\mathcal{C}_{k}$. We see on Figure 27(b) that the vertex $\widehat{v_{1}}$ corresponds to the vertex $v_{1}$ in the statement of Lemma 10.3. Hence, Lemma 10.3 ensures that $\widehat{v_{1}}$ is on $\mathcal{C}_{k}$ on the left of $\hat{v}$. We see on Figure 27(b) that there exists an oriented path $P$ going from $\widehat{v_{1}}$ to $\hat{v}$ such that each edge of $P$ leaves with label 1; but the last edge of $P$ is simply oriented, in contradiction with Lemma 10.7.

The case of Figure 27(c) can be treated similarly, as well as all configurations of 
Figure 26 .

Finally, Theorem 9.3 follows from Lemma 10.8 and from the fact that all possible configurations for a clockwise circuit of $X_{0}$ are listed in Figure 26.

\section{ACKNOWLEDGMENTS}

N. Bonichon and L. Castelli Aleardi are thanked for fruitful discussions.

\section{REFERENCES}

Alliez, P. And Gotsman, C. 2003. Recent advances in compression of 3D meshes. In Proc. of the Symp. on Multiresolution in Geometric Modeling. Cambridge. Available at http://www.inria.fr/rrrt/rr-4966.

Ambuørn, J., Biaeas, P., Burda, Z., Jurkiewicz, J., and Petersson, B. 1994. Sampling of random surfaces by baby universe surgery. Phys. Lett. B 325, 337-346.

di Battista, G., Tamassia, R., and Vismara, L. 1999. Output-sensitive reporting of disjoint paths. Algorithmica 23, 3, 302-340.

Bender, E. A. 1987. The number of three-dimensional convex polyhedra. Amer. Math. Monthly 94, 1, 7-21.

Bodirsky, M., GröPl, C., ANd KANG, M. 2003. Generating labeled planar graphs uniformly at random. In Proceedings of ICALP'03, Eindhoven, The Netherlands. LNCS. Springer Verlag, 1095-1107.

Bonichon, N., Felsner, S., And Mosbah, M. 2007. Convex drawings of 3-connected planar graphs. Algorithmica 47(4), 399-420.

Bonichon, N., Gavoille, C., And Hanusse, N. 2003. An information-theoretic upper bound of planar graphs using triangulations. In Proceedings of STACS'03, Berlin. LNCS. Springer Verlag, 499-510.

Bouttier, J., di Francesco, P., And Guitter, E. 2002. Census of planar maps: from the one-matrix solution to a combinatorial proof. Nucl. Phys. B 645, 477-499.

BREHM, E. 2000. 3-orientations and Schnyder 3-tree-decompositions. M.S. thesis, Freie Universität Berlin. http://www.tu-berlin.de/ felsner/Diplomarbeiten/brehm.ps.gz.

Brown, W. 1964. Enumeration of triangulations of the disk. Proceedings of the London Mathematical Society, 746-768.

Castelli-Aleardi, L. And Devillers, O. 2004. Canonical triangulation of a graph, with a coding application. 24pp, available at http://www.inria.fr/rrrt/rr-5231.html.

Castelli-Aleardi, L., Devillers, O., and Schaeffer, G. 2006. Optimal succinct representations of planar maps. In Proceedings of SoCG'06, Sedona (Arizona). ACM Press, 309-318.

Chuang, R. C.-N., Garg, A., He, X., KaO, M.-Y., and Lu, H.-I. 1998. Compact encodings of planar graphs via canonical orderings. In Proceedings of ICALP'g8, Aalborg, Denmark. LNCS. Springer Verlag, 118-129.

Denise, A., Vasconcellos, M., And Welsh, D. J. A. 1996. The random planar graph. Congr. Numer. 113, 61-79. Festschrift for C. St. J. A. Nash-Williams.

Duchon, P., Flajolet, P., Louchard, G., and Schaeffer, G. 2004. Boltzmann samplers for the random generation of combinatorial structures. Combinatorics, Probability and Computing 13,4-5, 577-625. Special issue on Analysis of Algorithms.

Felsner, S. 2001. Convex drawings of planar graphs and the order dimension of 3-polytopes. Order 18, 19-37.

Felsner, S. 2004. Lattice structures for planar graphs. Electron. J. Comb. 11, 1, Research paper R15, 24p.

Flajolet, P., Zimmermann, P., And Van Cutsem, B. 1994. A calculus for random generation of combinatorial structures. Theoret. Comput. Sci. 132, 2, 1-35.

de Fraysseix, H., Ossona de Mendez, P., and Rosenstiehl, P. Pigale, Automatic Graph Drawing. CAMS, EHESS, Paris. http://sourceforge.org/pigale/.

ACM Journal Name, Vol. V, No. N, Month 20YY. 
Fusy, É. 2005. Quadratic exact size and linear approximate size random generation of planar graphs. Discrete Mathematics and Theoretical Computer Science AD, 125-138.

Gessel, I. 1992. Super ballot numbers. J. Symbolic Comput. 14, 2/3, 179-194.

Gotsman, C. 2003. On the optimality of valence-based connectivity coding. Computer Graphics Forum, 99-102.

He, X., KaO, M.-Y., And Lu, H.-I. 1999. Linear-time succinct encodings of planar graphs via canonical orderings. SIAM J. on Disc. Math. 12, 3, 317-325.

He, X., KaO, M.-Y., And Lu, H.-I. 2000. A fast general methodology for informationtheoretically optimal encodings of graphs. SIAM J. Comput 30, 3, 838-846.

Kant, G. 1996. Drawing planar graphs using the canonical ordering. Algorithmica 16, 4-32. (also FOCS'g2).

Khodakovsky, A., Alliez, P., Desbrun, M., and Schröder, P. 2002. Near-optimal connectivity encoding of polygon meshes. Graphical Model 64, 3-4.

Khuller, S., Naor, J., And Klein, P. N. 1993. The lattice structure of flow in planar graphs. SIAM J. Discrete Math. 6(3), 477-490.

Lu, H.-I. 2002. Linear-time compression of bounded-genus graphs into information-theoretically optimal number of bits. In Proceedings of SODA'O2, San Francisco, California. ACM Press, 223-224.

McDiarmid, C., Steger, A., And Welsh, D. 2005. Random planar graphs. J. Combin. Theory, Series B 93, 187-205.

Mullin, R. And Schellenberg, P. 1968. The enumeration of c-nets via quadrangulations. J. Combin. Theory 4, 259-276.

Munro, J. I. and Raman, V. 1997. Succinct representation of balanced parentheses, static trees and planar graphs. In Proceedings of FOCS'97, Miami, Florida. ACM Press, 118-126.

Nijenhuis, A. And Wilf, H. S. 1978. Combinatorial Algorithms, Second ed. Academic Press.

Ossona de Mendez, P. 1994. Orientations bipolaires. Ph.D. thesis, Ecole des Hautes Etudes en Sciences Sociales, Paris.

Osthus, D., Prömel, H. J., and Taraz, A. 2003. On random planar graphs, their number and their triangulations. J. Combin. Theory Ser. B 88, 1, 119-134.

Poulalhon, D. and Schaeffer, G. 2006. Optimal coding and sampling of triangulations. Algorithmica 46(3-4), 505-527.

Rossignac, J. 1999. Edgebreaker: Connectivity compression for triangle meshes. IEEE Transactions on Visualization and Computer Graphics 5, 1, 47-61.

SchaEffER, G. 1997. Bijective census and random generation of Eulerian planar maps with prescribed vertex degrees. Electron. J. Combin. 4, 1, \# 20, 14 pp.

Schaeffer, G. 1999. Random sampling of large planar maps and convex polyhedra. In Proceedings of STOC'99, Atlanta. ACM Press, 760-769.

SChNyder, W. 1990. Embedding planar graphs on the grid. In Proceedings of SODA'90, San Francisco, California. ACM Press, 138-148.

Touma, C. and Gotsman, C. 1998. Triangle mesh compression. In Graphic Interface Conf. $26-34$.

Tutte, W. T. 1962. A census of planar triangulations. Canad. J. Math. 14, 21-38.

Tutte, W. T. 1963. A census of planar maps. Canad. J. Math. 15, 249-271.

Whitney, H. 1933. 2-isomorphic graphs. Amer. J. Math. 54, 245-254.

Wilson, D. B. 1997. Determinant algorithms for random planar structures. In Proceedings of SODA'97, New Orleans, Louisiana. ACM Press, 258-267.

Wilson, D. B. 2004. An annotated bibliography of perfectly random sampling with markov chains. Maintained on http://dimacs.rutgers.edu/ dbwils on/exact. 